\title{
Genomic imprinted genes in reciprocal hybrid endosperm of Brassica napus
}

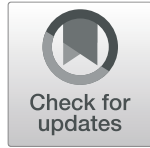

Hao Rong ${ }^{1}$, Wenjing Yang ${ }^{1}$, Haotian Zhu' ${ }^{1}$ Bo Jiang ${ }^{1}$, Jinjin Jiang ${ }^{1 *}$ and Youping Wang ${ }^{1,2^{*}}$

\begin{abstract}
Background: Genomic imprinting results in the expression of parent-of-origin-specific alleles in the offspring. Brassica napus is an oil crop with research values in polyploidization. Identification of imprinted genes in B. napus will enrich the knowledge of genomic imprinting in dicotyledon plants.

Results: In this study, we performed reciprocal crosses between B. napus L. cultivars Yangyou 6 (Y6) and Zhongshuang 11 (ZS11) to collect endosperm at 20 and 25 days after pollination (DAP) for RNA-seq. In total, we identified 297 imprinted genes, including 283 maternal expressed genes (MEGs) and 14 paternal expressed genes (PEGs) according to the SNPs between Y6 and ZS11. Only 36 genes (35 MEGs and 1 PEG) were continuously imprinted in 20 and 25 DAP endosperm. We found 15, 2, 5, 3, 10, and 25 imprinted genes in this study were also imprinted in Arabidopsis, rice, castor bean, maize, B. rapa, and other B. napus lines, respectively. Only 26 imprinted genes were specifically expressed in endosperm, while other genes were also expressed in root, stem, leaf and flower bud of B. napus. A total of 109 imprinted genes were clustered on rapeseed chromosomes. We found the LTR/Copia transposable elements (TEs) were most enriched in both upstream and downstream of the imprinted genes, and the TEs enriched around imprinted genes were more than non-imprinted genes. Moreover, the expression of 5 AGLs and 6 pectin-related genes in hybrid endosperm were significantly changed comparing with that in parent endosperm.

Conclusion: This research provided a comprehensive identification of imprinted genes in B. napus, and enriched the gene imprinting in dicotyledon plants, which would be useful in further researches on how gene imprinting regulates seed development.
\end{abstract}

\section{Background}

Genomic imprinting is an epigenetic regulation contrary to the classical Mendel's genetic law, which is reported mainly in plant endosperm and different tissues/organs in mammals [1-3]. Genes with parent-of-origin-specific expression or parentally biased expression are defined as imprinted genes $[4,5]$. Thus, the imprinted genes are classified into maternal expressed genes (MEGs) and paternal expressed genes (PEGs). Double fertilization is a specific phenomenon, involves two sperm cells from

\footnotetext{
* Correspondence: jjijang@yzu.edu.cn; wangyp@yzu.edu.cn

'Jiangsu Provincial Key Laboratory of Crop Genetics and Physiology, Yangzhou University, Yangzhou 225009, China

Full list of author information is available at the end of the article
}

male gametophyte which were fused with an egg cell and a homodiploid central cell to form a diploid zygote and triploid endosperm, respectively [6]. The triploid endosperm, a seed tissue that does not contribute genetic material to the offspring, has similar function as mammal placenta in providing nutrients for embryo development and seed formation. And it makes the parent-of-origin-specific effects more complicated because of the unbalanced contribution from maternal and paternal genomes [7-9]. Hitherto, the parent conflict theory is the primary explanation for the parent-oforigin-specific effects of imprinted genes on embryo development in plants, but gene imprinting is a

(c) The Author(s). 2021 Open Access This article is licensed under a Creative Commons Attribution 4.0 International License, which permits use, sharing, adaptation, distribution and reproduction in any medium or format, as long as you give appropriate credit to the original author(s) and the source, provide a link to the Creative Commons licence, and indicate if changes were made. The images or other third party material in this article are included in the article's Creative Commons licence, unless indicated otherwise in a credit line to the material. If material is not included in the article's Creative Commons licence and your intended use is not permitted by statutory regulation or exceeds the permitted use, you will need to obtain permission directly from the copyright holder. To view a copy of this licence, visit http://creativecommons.org/licenses/by/4.0/. The Creative Commons Public Domain Dedication waiver (http://creativecommons.org/publicdomain/zero/1.0/) applies to the data made available in this article, unless otherwise stated in a credit line to the data. 
complicated phenomenon that some imprinted loci need non-conflict theories $[10,11]$.

The first imprinted gene $R 1$ was discovered in maize through genetic trials [12]. But researches on plant imprinted genes were greatly lagged than that in mammals [6, 12-14]. Until 1999, imprinted genes in plants have been gradually identified by the analysis of transcript expressional level, reporter gene activity, or DNA methylation level on alleles that inherited from one specific parent following reciprocal crosses. These genes include MEA, FIS2, MPC, HDG3/8/9, FH5 in Arabidopsis, and FIE1, FIE2, PEG1, MEG1 in maize [9, 15-21]. The high-throughput sequencing technologies greatly facilitated the expression analysis of parental-derived alleles, thus more and more putative imprinted genes have been identified in plants, including Arabidopsis, wheat, rice, sorghum, Capsella rubella [22-29]. Although most imprinted genes are associated with seed development, but they are not conserved in different plants. Hitherto, a little percentage of imprinted genes were overlapped among different plant species [27, 29]. In addition, it has been proved that imprinted genes primarily influence the nutrient requirements and distribution during endosperm development through dosage effects, which are critical for seed development and vigor [30,31].

In mammals, approximately $80 \%$ of the imprinted genes are clustered on chromosomes, which can usually span millions of bases or more, and the expression of genes nearby can be regulated by these imprinting regulatory regions $[32,33]$. The clustered imprinted genes on chromosomes are often regulated by imprinting centers [4]. In plants, most of the imprinted genes are scattered on chromosomes, and only a small number of imprinted genes are clustered on chromosomes [24, 29, 34]. The number of clustered imprinted genes in plants was much less than that in mammals. As reported, the expression of imprinted genes is mainly regulated by DNA methylation and chromatin modification in both plants and mammals, and then the imprinted genes could regulate other gene expression by cis- or trans-regulation [7, 35]. However, it has also been reported that genomic DNA methylation was not associated with the expression of imprinted genes [29, 36]. Besides, transposable elements (TEs) have been taken as a primary driving force for genomic imprinting, and TEs are extensively identified with hypomethylation on the genome of endosperm, which is important to the seed development $[27,36,37]$. TEs are a large number of repetitive elements on the genome of eukaryotes, which play an important role in plant genome evolution and structural changes [38]. TEs could be activated during hybridization between species and introgression of foreign fragments, and then regulate the downstream gene expression [39]. In castor bean, two types of TEs (LTR/Gypsy and LTR/Copia) were significantly enriched in the vicinity of imprinted genes, and these TEs were extensively demethylated during seed development [27]. Thus, it is speculated that the methylation status of LTR/Gypsy and LTR/Copia TEs might be the main cause of gene imprinting. But this phenomenon was not observed in Arabidopsis and maize, which might be due to the specific TE distribution in different plant species [26, 34]. In A. thaliana, repression of AGAMOUS-LIKE MADS-box genes (AGLS) in mutants of imprinted genes were related to the abnormal endosperm cellularization. Down-regulation of genes involved in carbohydrate metabolism (especially genes encode polygalacturonases) would affect pectin hydrolysis in triploid mutants of $A$. thaliana imprinted genes, and finally influence the endosperm cellularization and seed viability [40].

The third largest oil crop in the world, Brassica napus L. (AACC, $2 \mathrm{n}=38$ ), is an allopolyploid derived from natural hybridization between two diploids $B$. rapa (AA, $2 \mathrm{n}=20)$ and B. oleracea $(C C, 2 \mathrm{n}=18)$ [41]. Since it is a major resource of edible oil, biofuel, and animal fodder, genetic and epigenetic researches on $B$. napus are important to its breeding course [42]. Comprehensive identification of imprinted gene in B. napus will be helpful to elucidate the genetic regulation of seed development. Since the cellularized endosperm in B. napus does not proliferate as in monocotyledon crops (e.g. maize, wheat, rice) and castor bean, but gradually disappeared with seed development. It is challenging to collect rapeseed endosperm for genome imprinting analysis [43, 44]. Hitherto, genome imprinting was barely reported in Brassica, except for two studies on B. rapa and B. napus, which identified the putative imprinted genes at one developmental stage of endosperm $[45,46]$. Thus, identification of imprinted genes from the reciprocal endosperm of $B$. napus, including different developmental stages, will be of great benefit to the genetic mechanism of genomic imprinting in dicotyledon plants.

In the present study, we performed high-throughput RNA-seq on the endosperm of reciprocal crosses between two B. napus cultivars, Zhongshuang 11 (ZS11) and Yangyou 6 (Y6). The maternal and paternal specific single nucleotide polymorphisms (SNPs) were identified for genome-wide screening of imprinted genes. Based on the parent-specific expression, we identified 297 imprinted genes, including 283 MEGs and 14 PEGs. Interestingly, 36 of 297 imprinted genes were continuously imprinted during endosperm development. Only 26 imprinted genes were specifically expressed in endosperm, while other genes were also expressed in other tissues of B. napus, rather than endosperm-specific. A total of 109 imprinted genes were clustered on rapeseed chromosomes, and we found the LTR/Copia TEs were most enriched in both upstream and downstream of the 
imprinted genes. This research provided a more comprehensive identification of imprinted genes in B. napus, and enriched the gene imprinting in dicotyledon plants, which would be useful in further researches on how gene imprinting regulates seed development.

\section{Results \\ Transcriptome sequencing and parental specific SNP calling}

To distinguish the parental-derived allelic expression in hybrids, we performed the deep high-throughput RNA sequencing on the 20 days after pollination (DAP) and 25 DAP endosperm of ZS11 and Y6 to discover the SNPs between two parents. In total, we obtained 399.78 million paired-end reads, with an average of 35.33, 30.2, 35.46 and 31.97 million reads for 20 DAP endosperm of ZS11 (ZS11_20 DAP), 25 DAP endosperm of ZS11 (ZS11_25 DAP), 20 DAP endosperm of Y6 (Y6_20 DAP), and 25 DAP endosperm of Y6 (Y6_25 DAP), respectively. These reads were mapped to $61,278,60$, $174,60,604$ and 59,801 genes of $B$. napus with fragments per kilobase per million (FPKM) $>1$ in the endosperm of ZS11_20 DAP, ZS11_25 DAP, Y6_20 DAP, Y6_25 DAP, respectively. The three biological replicates of RNA-seq data were confirmed with personal correlation coefficient values of $R=0.96 \sim 0.99$ (Additional file 1: Fig. S1). The SNPs of ZS11 and Y6 were identified in comparison to the B. napus (European winter oilseed cultivar 'Darmor-bzh') reference genome using Hisat2 and Samtools. Based on the 91.22, 93.03, 92.95 and $92.96 \%$ reads in ZS11_20 DAP, ZS11_25 DAP, Y6_20 DAP, and Y6_25 DAP mapped to the reference genome, the uniquely mapped reads were retained for stringent SNP screening, only the homozygous SNPs identified in at least two biological replicates and covered by $\geq 10$ reads in each sequencing library were kept for further analysis. A total of 35,928 and 28,775 SNPs between ZS11 and Y6 were identified in $20 \mathrm{DAP}$ and 25 DAP endosperms, respectively. These SNPs covered 15,738 and 13,721 genes, of which, 10,672 genes were overlapped in the 20 DAP and 25 DAP endosperm (Additional file 2: Table S1; Additional file 3: Table S2). Similarly, the uniquely mapped reads in the endosperm of reciprocal hybrids (7.55 and 5.57 million reads of 20 and 25 DAP hybrid endosperm) were used for SNP identification, and the reads with paternal or maternal specific SNPs were extracted for allelic expression analysis (Additional file 4: Table S3; Additional file 5: Table S4).

\section{Genome-wide identification of imprinted genes in hybrid endosperm}

Based on the ratio of maternal-derived and paternalderived reads at each SNP loci, we used stringent criteria to screen the imprinted genes that MEGs should obey to allelic expression ratio of maternal: paternal $\geq 10$ : 1 (5 times of maternal: paternal $=2: 1, \geq 90 \%$ maternally biased expression), and PEGs were screened with a ratio of paternal: maternal $\geq 3: 2$ (3 times of paternal: maternal $=1: 2, \geq 60 \%$ paternally biased expression) in both reciprocal hybrid endosperm (Additional file 4: Table S3; Additional file 5: Table S4). The genes with parental biased expression in three biological replicates of reciprocal hybrid endosperm were identified as imprinted genes $(q<0.05)$. We found that the majority of these genes obeyed the ratio of maternal: paternal $=2$ : 1 in both 20 DAP and 25 DAP endosperm, only $1.132 \%$ genes exhibited parent-of-origin differences in allelic expression ( $x^{2}$ test, $q<0.05$ ) (Fig. 1a). In total, we identified 251 imprinted genes (242 MEGs and 9 PEGs) in 20 DAP endosperm and 82 imprinted genes (76 MEGs and 6 PEGs) in 25 DAP endosperm (Additional file 6: Table S5; Additional file 7: Table S6). Only 36 genes (35 MEGs and 1 PEG) were continuously imprinted in 20 and 25 DAP endosperm. The remaining 261 genes were imprinted in different stages of endosperms, including 215 genes imprinted in 20 DAP endosperm (207 MEGs and 8 PEGs) and 46 genes imprinted in 25 DAP endosperm (41 MEGs and 5 PEGs) (Fig. 1b; Additional file 8: Table S7). This indicated that most imprinted genes exhibited inconsistent expression pattern at different developmental stages of endosperm in B. napus. Among the stage specific imprinted genes, 73 genes were imprinted in one stage but exhibited bi-allelic expression pattern in another developmental stage of hybrid endosperm. The rest 118 genes were imprinted in only one stage, or imprinted in two developmental stages but supported by less than 10 reads in one stage. Moreover, we found 8 pairs of homologous genes imprinted in A and C subgenome (BnaA05g00640D and BnaC04g51420D, BnaA06g07630D and BnaC05g09100D, BnaA10g22530D and BnaC03g49920D, BnaA08g18690D and BnaC03g58190D, BnaA03g21790D and BnaC03g26060D, BnaA10g19500D and BnaC09g54550D, BnaA09g42740D and BnaC08g35220D, BnaA01g23480D and BnaCnng44170D). As to the remaining 281 unpaired genes, 200 genes (71\%) were imprinted on the A subgenome and 81 genes (29\%) were imprinted on the C subgenome. This indicated that most of the imprinted genes were biased to A subgenome.

\section{Experimental validation of candidate imprinted genes in B. napus}

In order to confirm the imprinted genes in B. napus, the allele-specific expression analysis was carried out on randomly selected genes (7 MEGs and 1 PEG). The amplified RT-PCR fragments of endosperms from reciprocal crosses and self-pollinated parents were sequenced to identify the parental SNPs. Consistent with the SNPs 

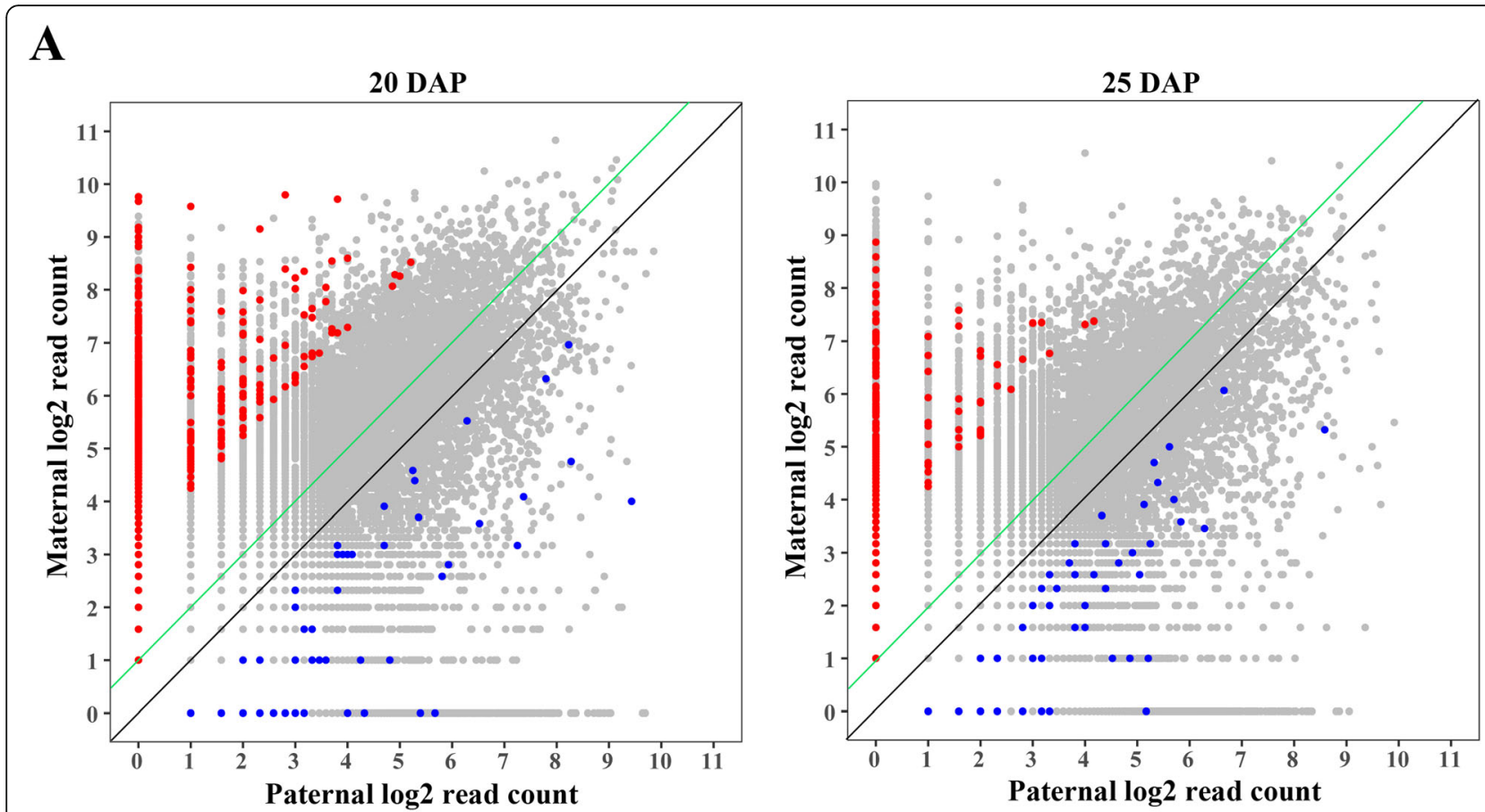

B
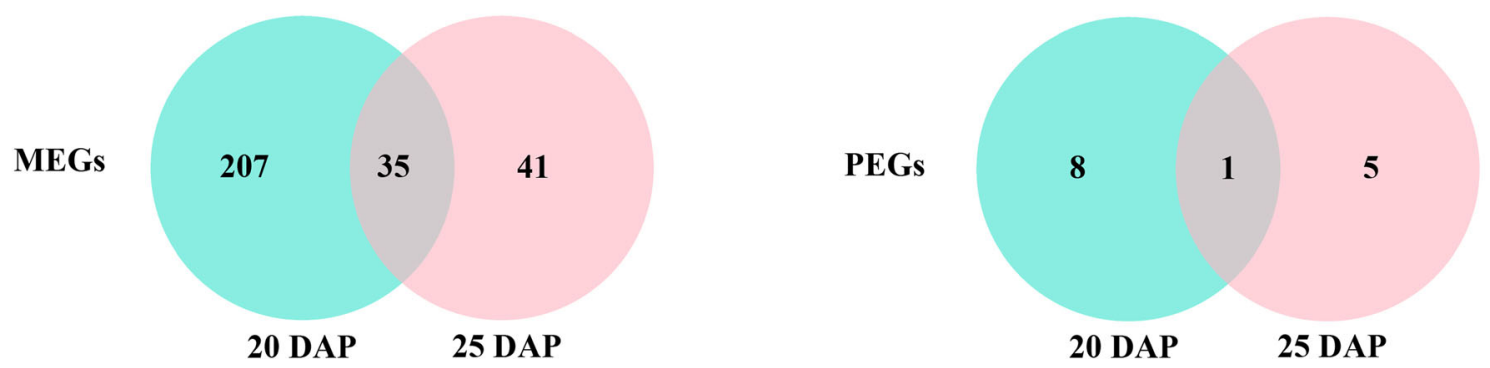

Fig. 1 Allele-specific expression analysis of genes in reciprocal endosperm. a. $\log _{2}$ normalized read counts for all SNP loci for the endosperms. The red dots represent the MEG loci sorted out with $>90 \%$ read percentage bias and the blue dots represent the PEG loci sorted out with $>60 \%$ read percentage bias in both reciprocal cross endosperms $(q<0.05)$. The black line and green line denoted the 1:1 and 2:1 ratios (maternal: paternal), respectively. b. Venn diagram of MEGs and PEGs between 20 and 25 DAP endosperm. MEG, maternal expressed gene. PEG, paternal expressed gene. DAP, day after pollination

identified by RNA-seq data, all the 8 genes were confirmed with ZS11 or Y6 specific SNPs in the hybrid endosperm, indicating these genes with parent-of-origin expression patterns (Fig. 2). For example, the MEGs (BnaA02g11050D, BnaA04g15380D, BnaA05g04610D, BnaA09g52990D, BnaC02g10080D, BnaC08g30150D, BnaCnng51340D) were identified with maternal specific SNPs in both reciprocal crosses. While a PEG (BnaA04g19090D) was confirmed with paternal specific SNP in both reciprocal crosses.

To know whether these imprinted genes identified in $B$. napus were conserved in other plant species, we blasted their homologous gene pairs in other species, and found 15, 2, 5, 3, 10, and 25 imprinted genes in this study were also imprinted in Arabidopsis [22, 23], rice [24], castor bean [27], maize [25], B. rapa [45], and other
B. napus lines [46], respectively (Table 1). Interestingly, most of the overlapped genes were MEGs (except for three PEGs overlapped with $B$. rapa), and the imprinted genes in $B$. napus were more conserved with other dicots than monocots. We found the homologs of BnaA06g38220D were also imprinted in rice and maize. This agreed to the previous report that gene imprinting is poorly conserved among different species [27].

\section{Functional characterization of imprinted genes in $B$. napus}

As reported in other plants, imprinted genes were mostly identified in endosperm, and with endospermspecific or endosperm-preferred expression patterns [22, $24,47]$. Here, we analyzed the expression pattern of rapeseed imprinted genes in different tissues of $B$. napus 


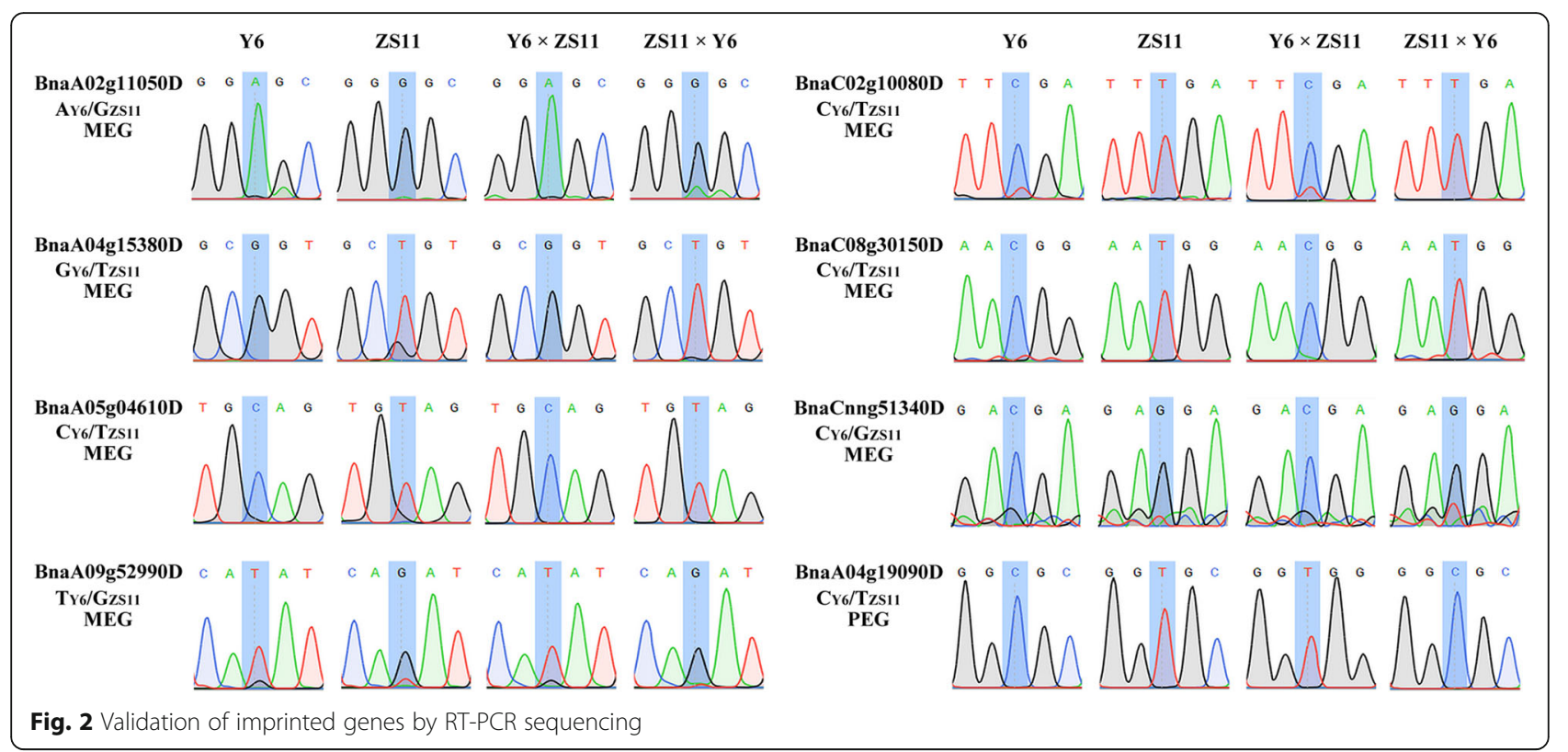

cv. ZS11, including root, stem, leaf, bud and endosperm (Fig. 3a; Additional file 9: Table S8). Inconsistent with previous reports, we only found 26 rapeseed imprinted genes were specifically expressed in endosperm. While other imprinted genes were also expressed in root, stem, leaf and flower bud of B. napus, rather than endospermspecific. Thus, we may suspect that most of the imprinted genes have functions in the development of rapeseed, not only with specific effects on the endosperm development. In addition, we also compared the expression of imprinted genes and non-imprinted genes in the parent endosperm. The results showed that the imprinted genes were highly expressed than nonimprinted genes in both 20 DAP and 25 DAP endosperm. Besides, the expression of MEGs in parent endosperm was higher than that of PEGs (Fig. 3b).

In mammalian, most of the imprinted genes are clustered on the chromosomes, and their expression is regulated by imprinted regulatory regions [48]. But in plants (e.g. Arabidopsis, rice, sorghum, and castor bean), only a few imprinted genes are clustered on the chromosome. Here, we mapped 245 imprinted genes (except for 47 MEGs and 5 PEGs scattered to Ann_random or Cnn random chromosomes) to the 19 chromosomes of $B$. napus. Altogether, we identified 35 clusters that unevenly distributed on the $\mathrm{A}$ and $\mathrm{C}$ subgenome (Fig. 4; Additional file 10: Table S9), including 29 clusters (92 imprinted genes) on A genome and 6 clusters (17 imprinted genes) on $\mathrm{C}$ genome. These clustered genes might be controlled by regional regulations. Besides, we found five largest clusters, including cluster 16 (5 imprinted genes) on the end of A05, cluster 24 (5 genes) and cluster 25 (6 genes) on A08, cluster 28 (5 genes) and cluster 29 (5 genes) on A10. Kyoto Encyclopedia of Genes and Genomes (KEGG) analysis on genes nearby the cluster 24/25/28/29 revealed that most genes were involved in transcription, translation, energy metabolism, glycan biosynthesis and metabolism, transport and catabolism, plant hormone signal transduction, and environmental adaptation (Fig. 5). Gene ontology (GO) enrichment analysis revealed that most imprinted genes were assigned to biological functions (cellular process, single-organism process, metabolic process, response to stimulus and biological regulation), molecular functions (binding and catalytic activity), cellular components (cell and cell part) (Fig. 6a; Additional file 11: Table S10). In triploid $a d m$ and suvh 7 mutants of A. thaliana, the seed rescue was strongly correlated with decreased expression of AGLs, which may further affect endosperm cellularization and cause embryo arrest. Suppression of genes related to pectin hydrolysis in these mutants were also related to the abnormal endosperm cellularization and seed viability [40]. Among the top enriched GO terms, we found galacturonate biosynthetic and metabolic process, cell wall polysaccharide and macromolecule metabolic process, UDP-glucuronate 4-epimerase activity, and alpha-(1,2)-fucosyltransferase activity were enriched (Fig. 6b, c). This indicated that many imprinted genes were involved in the cell wall biosynthesis and related to endosperm cellularization. In rapeseed, endosperm accumulated and cellularized until 18 to 32 DAP, which was then disappeared after transferring nutrients to embryos [43]. Thus, imprinting of these genes related to cell wall biosynthesis would affect pectin hydrolysis, endosperm cellularization and seed viability. Further molecular functional studies on these 
Table 1 Overlaps between the rapeseed imprinted genes and those of Arabidopsis, rice, castor bean, maize, and other Brassicas

\begin{tabular}{|c|c|c|c|c|c|c|c|}
\hline & Query & Subject & Identity & Mapping length & Mismatch & Gap & E value \\
\hline \multirow[t]{15}{*}{ Arabidopsis } & BnaA01g15540D & AT4G26420 & 76.47 & 652 & 90 & 6 & 0 \\
\hline & BnaA03g27380D & AT3G01640 & 90.29 & 264 & 95 & 3 & 1.00E-110 \\
\hline & BnaA03g42520D & AT4G16380 & 75.67 & 248 & 20 & 1 & $6.00 \mathrm{E}-148$ \\
\hline & BnaA04g19750D & AT2G33770 & 81.26 & 122 & 59 & 1 & $2.00 \mathrm{E}-36$ \\
\hline & BnaA05g08350D & AT2G35670 & 46.67 & 744 & 264 & 9 & 0 \\
\hline & BnaA05g27940D & AT3G11000 & 56 & 547 & 42 & 3 & 0 \\
\hline & BnaA06g05440D & AT1G09540 & 65.96 & 290 & 23 & 1 & 0 \\
\hline & BnaA07g33830D & AT1G77960 & 38.12 & 669 & 308 & 10 & $7.00 \mathrm{E}-178$ \\
\hline & BnaA08g26320D & AT1G09540 & 83.15 & 629 & 47 & 3 & 0 \\
\hline & BnaA09g01980D & AT3G27300 & 92.25 & 580 & 49 & 2 & 0 \\
\hline & BnaA10g22530D & AT5G64260 & 78.43 & 306 & 30 & 1 & 5.00E-162 \\
\hline & BnaC05g48510D & AT3G02110 & 90.7 & 473 & 32 & 2 & 0 \\
\hline & BnaC07g22140D & AT2G03210 & 86 & 313 & 33 & 0 & 0 \\
\hline & BnaC09g20470D & AT2G01300 & 82.05 & 422 & 30 & 1 & 0 \\
\hline & BnaCnng 402100 & AT3G16720 & 68.52 & 578 & 191 & 9 & 0 \\
\hline \multirow[t]{10}{*}{ B. rapa } & BnaA01g08850D & Bra013274 & 85.08 & 496 & 46 & 2 & 0 \\
\hline & BnaA04g19090D & Bra005543 & 57.7 & 461 & 84 & 2 & $2 \mathrm{E}-123$ \\
\hline & BnaA05g08350D & Bra005316 & 50.26 & 191 & 14 & 4 & $4 \mathrm{E}-47$ \\
\hline & BnaA05g08770D & Bra005362 & 59.74 & 688 & 201 & 1 & 0 \\
\hline & BnaA06g05440D & Bra020016 & 84.77 & 243 & 14 & 0 & $2 \mathrm{E}-108$ \\
\hline & BnaA06g37970D & Bra019964 & 87.5 & 392 & 22 & 1 & 0 \\
\hline & BnaA08g26320D & Bra020016 & 82.34 & 368 & 24 & 3 & $2 \mathrm{E}-151$ \\
\hline & BnaA09g13800D & Bra027091 & 65.84 & 363 & 17 & 5 & $3 E-109$ \\
\hline & BnaAnng03730D & Bra036061 & 82.27 & 141 & 8 & 1 & $7 \mathrm{E}-62$ \\
\hline & BnaC02g44940D & Bra007859 & 78.81 & 387 & 32 & 3 & 0 \\
\hline \multirow[t]{2}{*}{ Rice } & BnaA06g38220D & LOC_Os03g01320.1 & 76.19 & 84 & 20 & 0 & $7 E-39$ \\
\hline & BnaA09g13800D & LOC_Os07g37620.1 & 49.03 & 155 & 63 & 5 & $7 E-33$ \\
\hline \multirow[t]{5}{*}{ Castor bean } & BnaA06g34330D & $29,780 \cdot m 001362$ & 68.1 & 232 & 45 & 3 & $5 E-109$ \\
\hline & BnaC05g09100D & $29,792 . m 000624$ & 50.21 & 482 & 234 & 5 & $2 \mathrm{E}-174$ \\
\hline & BnaA08g21920D & $30,170 \cdot m 014165$ & 46.04 & 556 & 210 & 17 & $3 E-108$ \\
\hline & BnaC04g34200D & $29,905 \cdot m 000439$ & 39.93 & 278 & 149 & 7 & $3 \mathrm{E}-50$ \\
\hline & BnaC07g36310D & 27,837.m000165 & 66.43 & 143 & 48 & 0 & $1 \mathrm{E}-42$ \\
\hline \multirow[t]{3}{*}{ Maize } & BnaA06g38220D & GRMZM2G379898 & 74.12 & 85 & 22 & 0 & $4 \mathrm{E}-38$ \\
\hline & BnaA04g15380D & GRMZM2G445602 & 70.26 & 548 & 142 & 5 & 0 \\
\hline & BnaA08g16960D & GRMZM2G108032 & 56.93 & 476 & 199 & 3 & 0 \\
\hline \multirow[t]{9}{*}{ B. napus(Rapeseed lines YN171 and 93275) } & BnaA02g15580D & $B n A 02 g 0069250$ & 99.02 & 509 & 5 & 0 & 0 \\
\hline & BnaA02g34950D & $B n A 02 g 0050760$ & 99.42 & 344 & 2 & 0 & 0 \\
\hline & BnaA03g24210D & BnA09g0353510 & 93.07 & 231 & 15 & 1 & $1 \mathrm{E}-156$ \\
\hline & BnaA03g31730D & BnC03g0579390 & 85.71 & 385 & 53 & 1 & 0 \\
\hline & BnaA03g48600D & $B n A 03 g 0151360$ & 100 & 173 & 0 & 0 & $4 \mathrm{E}-125$ \\
\hline & BnaA04g24790D & $B n A 04 g 0183470$ & 99.03 & 516 & 5 & 0 & 0 \\
\hline & BnaA06g07630D & BnA06g0231270 & 98.8 & 502 & 6 & 0 & 0 \\
\hline & BnaA06g26050D & $B n A 06 g 0253460$ & 100 & 405 & 0 & 0 & 0 \\
\hline & BnaA07g18120D & BnA03g0127550 & 99.61 & 507 & 2 & 0 & 0 \\
\hline
\end{tabular}


Table 1 Overlaps between the rapeseed imprinted genes and those of Arabidopsis, rice, castor bean, maize, and other Brassicas (Continued)

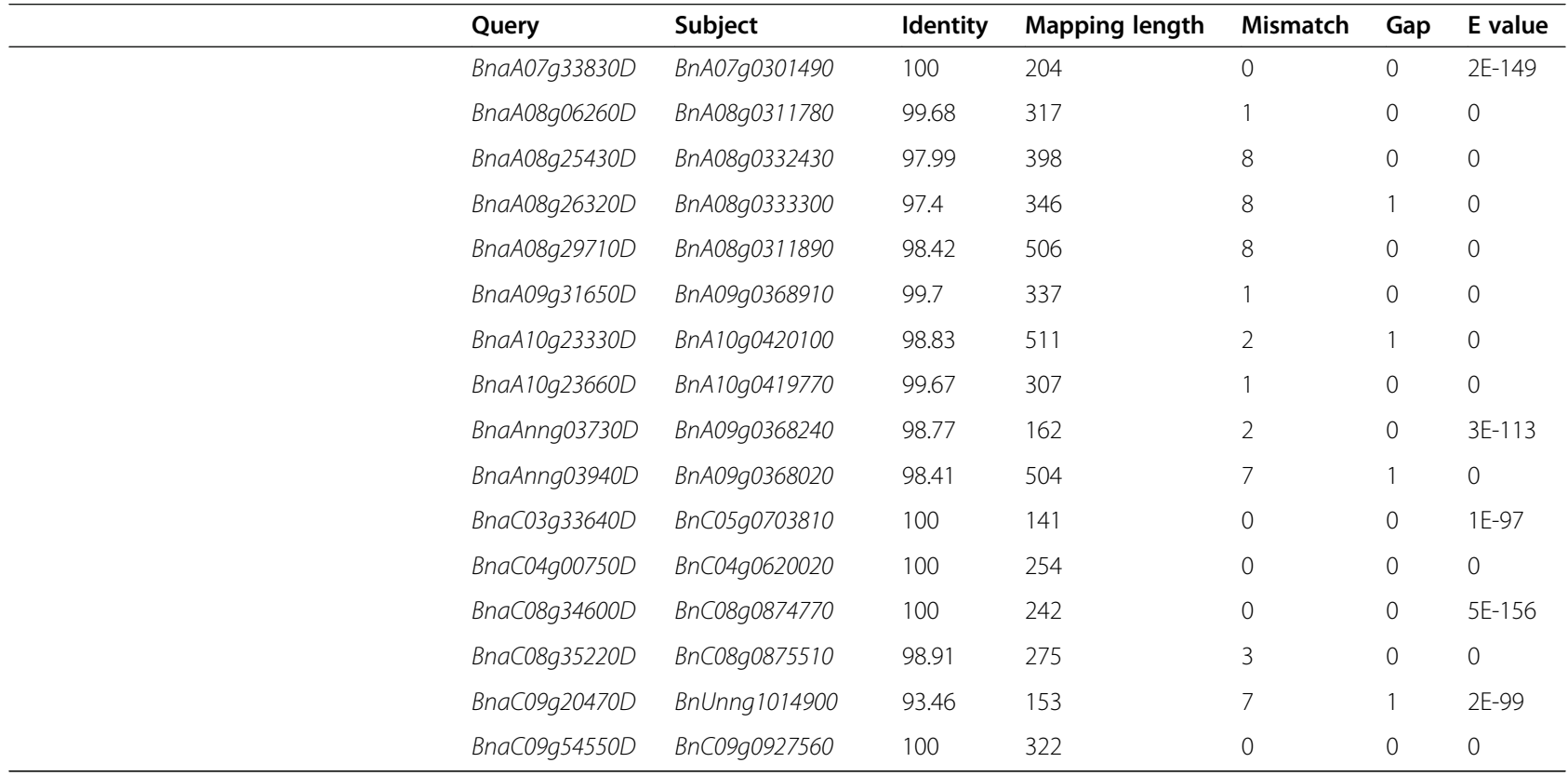

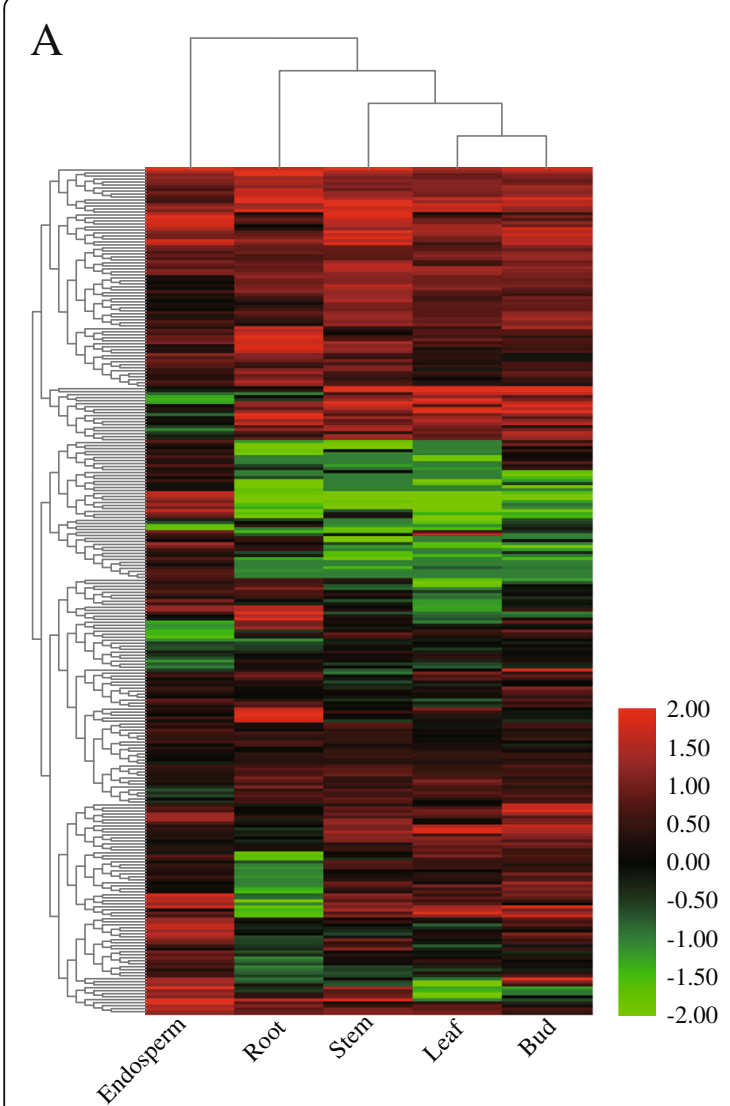

B

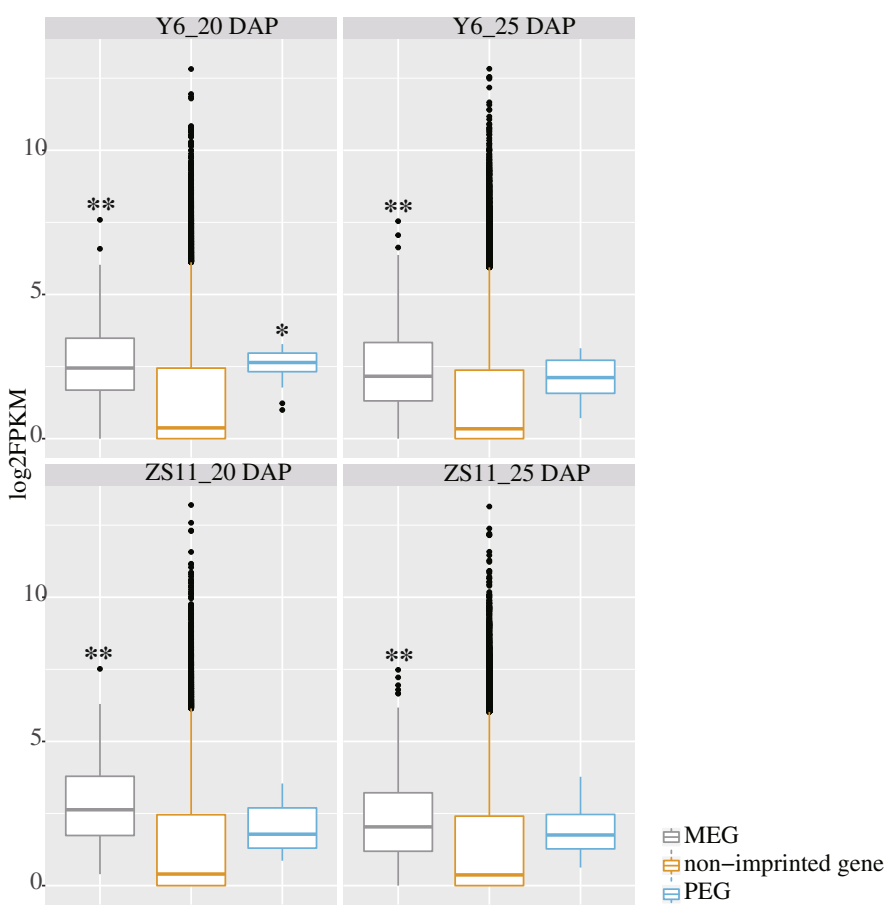

Fig. 3 Expression pattern of imprinted genes. a. Heat map of imprinted genes in endosperm, root, stem, leaf and bud ( $\log _{10}$ FPKM). b. Box-plot of expression of imprinted genes and non-imprinted genes in 20 DAP and 25 DAP endosperm of parents ( $\left.\log _{2} F P K M\right)$. Student's $t$-test was used for statistical analysis between imprinted genes and non-imprinted genes $\left({ }^{*}, p \leq 0.05 ;{ }^{* *}, p \leq 0.01\right)$ 


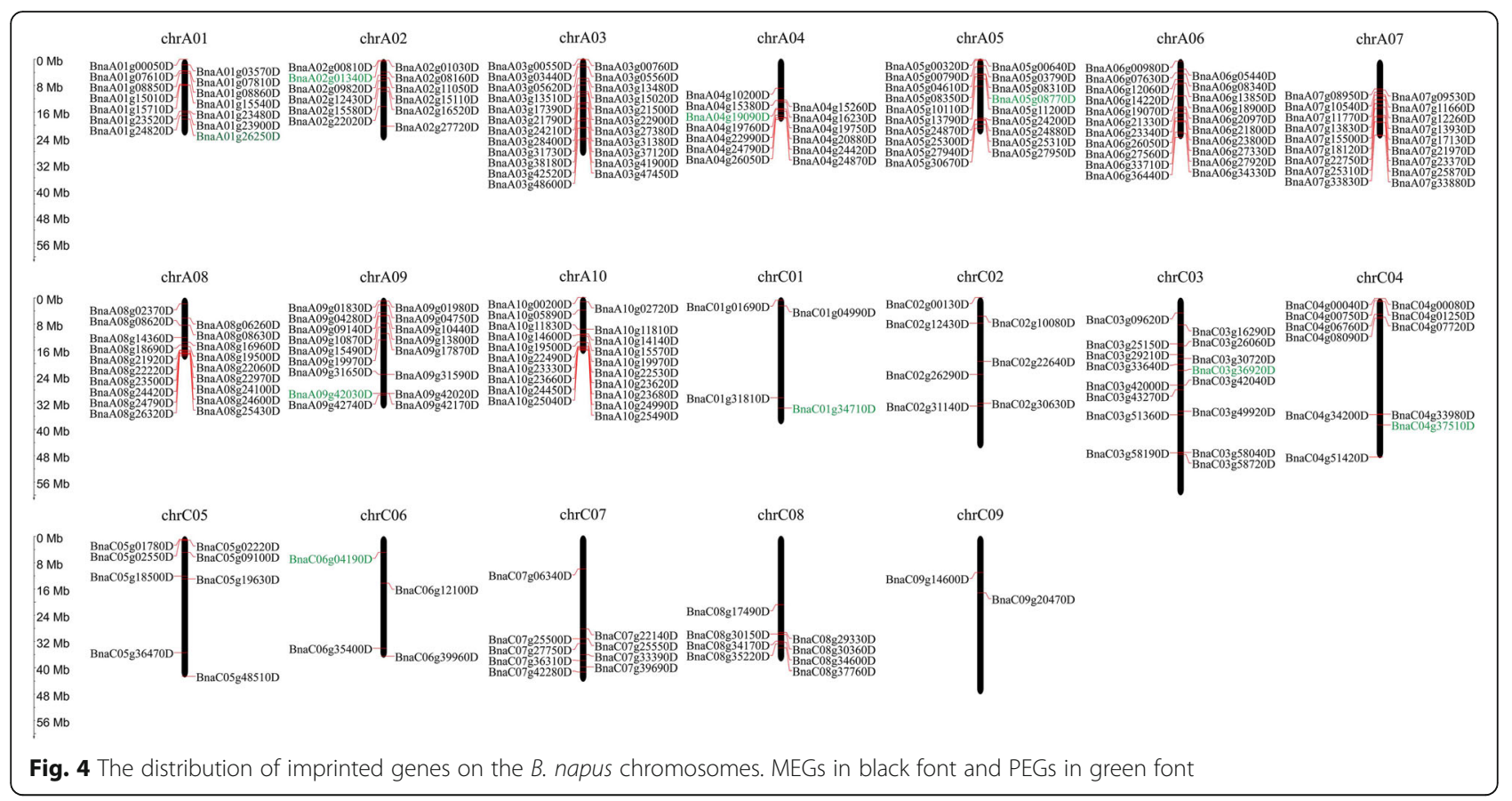

imprinted genes would enrich our knowledge in seed development of B. napus.

\section{Transposable element enrichment around imprinted genes}

In Arabidopsis endosperm, the extensively demethylated TEs have been taken as a main driving force of gene imprinting $[36,49]$. Therefore, we investigated the number and type of TEs within $5 \mathrm{~kb}$ upstream and downstream of the imprinted and non-imprinted genes in B. napus. We found the number of TEs enriched around imprinted genes was significantly higher than that around non-imprinted genes. And the TE types enriched in $5^{\prime}$-region were more than that in $3^{\prime}$-region of the imprinted gene (Fig. 7a). Interestingly, we found the LTR/Copia TEs were enriched in both upstream and downstream of the imprinted genes. This was similar to the TE enrichment around imprinted genes in castor bean, which were enriched with LTR/Copia and LTR/Gypsy type TEs. But DNA/MuDR type TEs were enriched around imprinted genes in Arabidopsis and C. rubella, while CACTA type TEs were enriched around imprinted genes in maize [26-28, 34]. In addition, we analyzed the imprinted genes with TEs enriched in both $5^{\prime}$ - and $3^{\prime}$-regions. We found 5' - and $3^{\prime}$ region of BnaA01g08860D was enriched with 5 and 2 TEs,
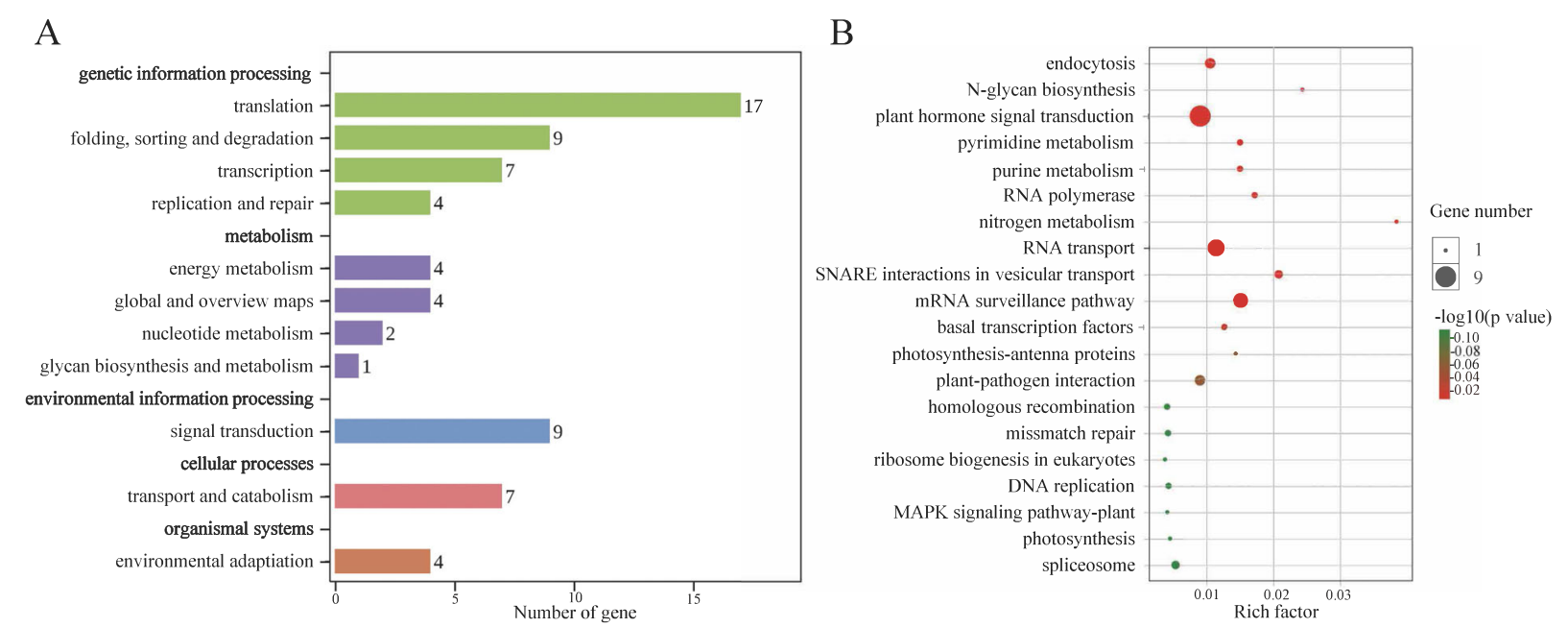

Fig. 5 KEGG enrichment of imprinted clusters. a. KEGG pathway analysis of genes within the cluster 24/25/28/29. b. Top 20 pathways enriched with genes in the cluster 24/25/28/29. Rich factor means the ratio of imprinted gene number to transcript number in each KEGG pathway 


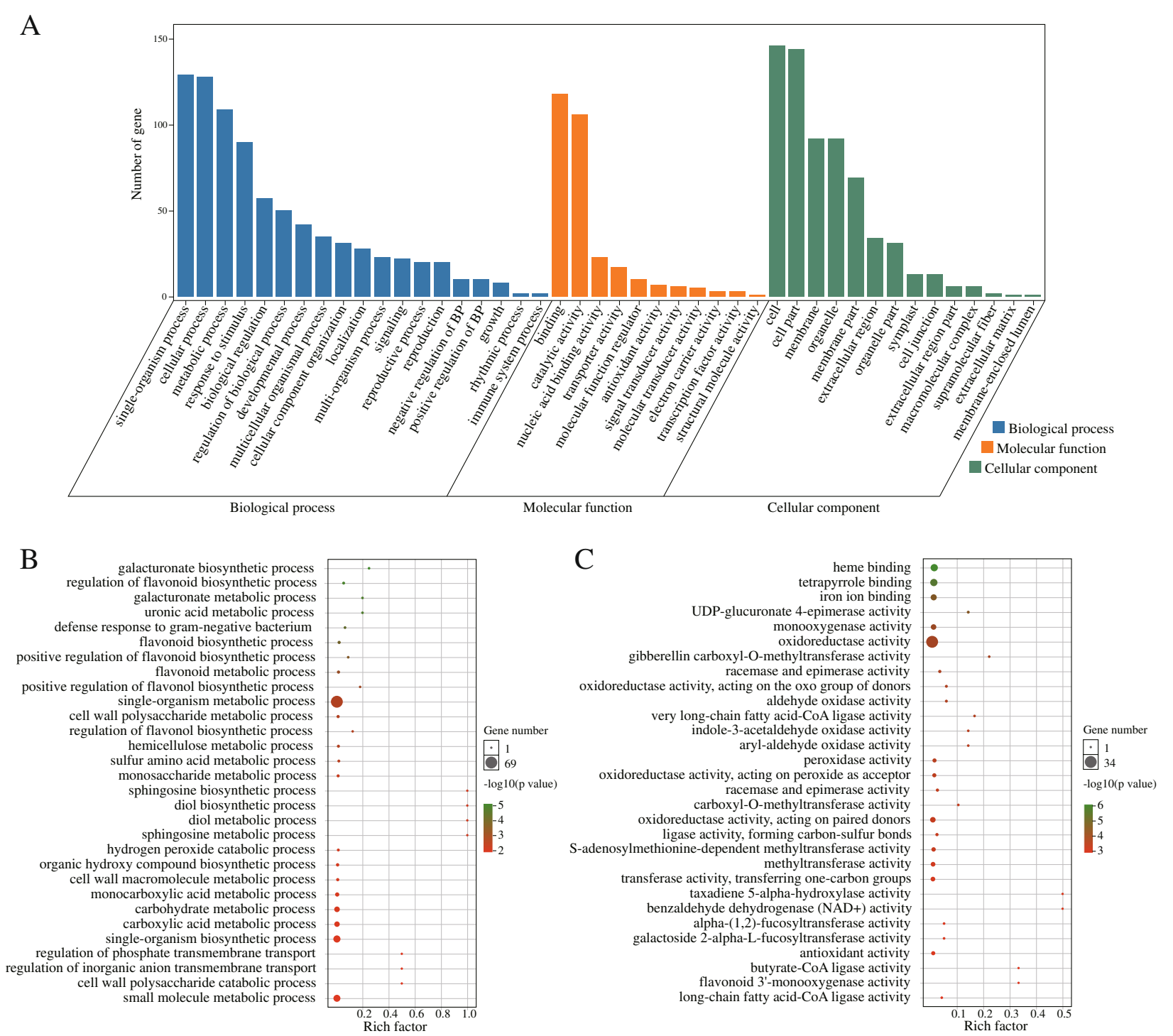

Fig. $6 \mathrm{GO}$ enrichment analysis. a. GO enrichment analysis of imprinted genes. b. Top $30 \mathrm{GO}$ terms enriched in biological process. c. Top $30 \mathrm{GO}$ terms enriched in molecular function. Rich factor means the ratio of imprinted gene number to transcript number in each GO term

respectively. The expression level of BnaA01g08860D in endosperm was much lower than that in other tissues. The 5'-region of BnaA08g29710D and BnaA04g10200D contain 2 and 3 TEs, and the $3^{\prime}$-region contain 1 and 2 TEs, respectively. BnaA08g29710D and BnaA04g10200D were highly expressed in endosperm than in other tissues (Fig. 7b, c). We suspected that the TEs enriched around these imprinted genes might affect their expression pattern.

\section{Expression analysis of $A G L s$ and genes related to pectin degradation}

As reported in A. thaliana, the expression level of $A G L s$ and genes involved in carbohydrate metabolism (e.g. genes encode polygalacturonases) affected endosperm cellularization and pectin hydrolysis in triploid mutants of $A$. thaliana imprinted genes $[40,50]$. Since some imprinted genes in rapeseed were also enriched with GO terms related to cell wall synthesis, here we analyzed the expression of 113 AGLs and the 189 genes involved in pectin degradation pathway in the reciprocal hybrid endosperm (Fig. 8). Comparing with two parents, we found the expression of 5 AGLs (BnaC02g01970D, BnaA03g29530D, BnaA06g12900D, BnaC02g40410D and BnaC01g28010D) were changed with $\mid \log _{2}$ fold change $\mid>5$ in hybrid endosperm, and the expression of most $A G L s$ were not significantly changed. As to the genes involved in pectin degradation pathway, we found the expression of BnaC08g30060D, BnaA09g16050D, BnaC04g24110D, BnaA01g04630D, BnaC01g06140D and BnaA06g16310D were significantly changed in hybrid endosperm compared with that in parent endosperm. 


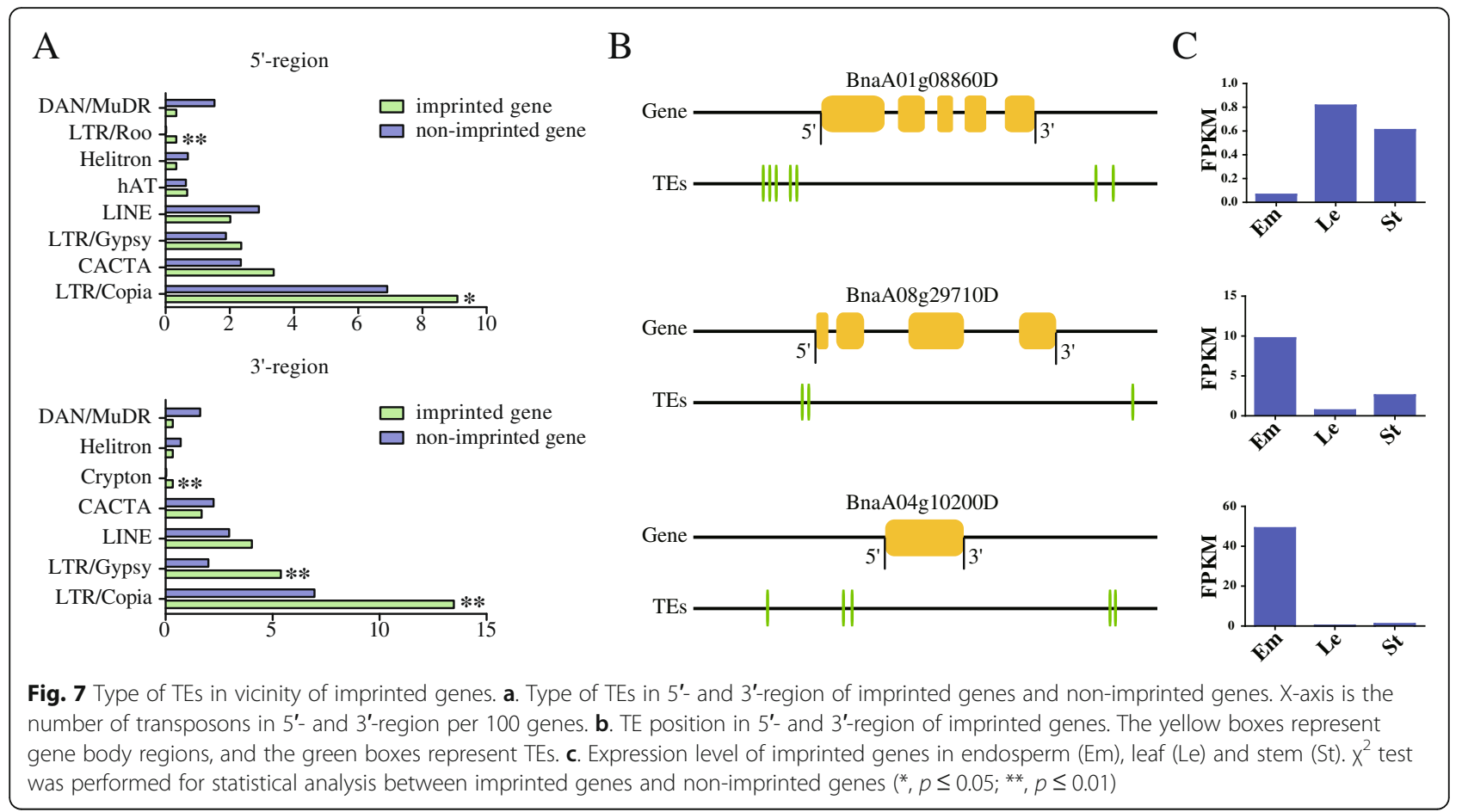

\section{Discussion}

Genomic imprinting is an epigenetic phenomenon against to the classical Mendel's law. Hitherto, imprinted genes have been identified in several plants, including Arabidopsis (341 genes), rice (262 genes), maize (356 genes), castor bean (209 genes), and wheat (372 genes) $[23-25,27,47]$. Functional annotation of these imprinted genes revealed that they are mainly enriched in the regulation of pigmentation, protein storage, transcriptional regulation, catalysis and chromatin modification [26]. However, the functional studies on imprinted genes are barely reported, which should be crucial for genetic regulation on plant development, especially seed development $[15,51-53]$. B. napus is the third largest oil crop with great economic values, but only one report on genome imprinting of 30 DAP endosperm is available now [46]. Research on gene imprinting of different $B$. napus cultivars will broaden our understanding of seed development, and lay a basis for genetic breeding of rapeseed with high-yield and high-quality.

\section{Genomic imprinting pattern in endosperm of $B$. napus}

In this study, we generated transcriptome sequencing data of developing endosperm from two B. napus cultivars (Y6 and ZS11) and their reciprocal hybrids. Based on a stringent criteria for screening of genes with paternal or maternal-specific SNPs, we identified 297 imprinted genes in hybrid endosperm, including 283
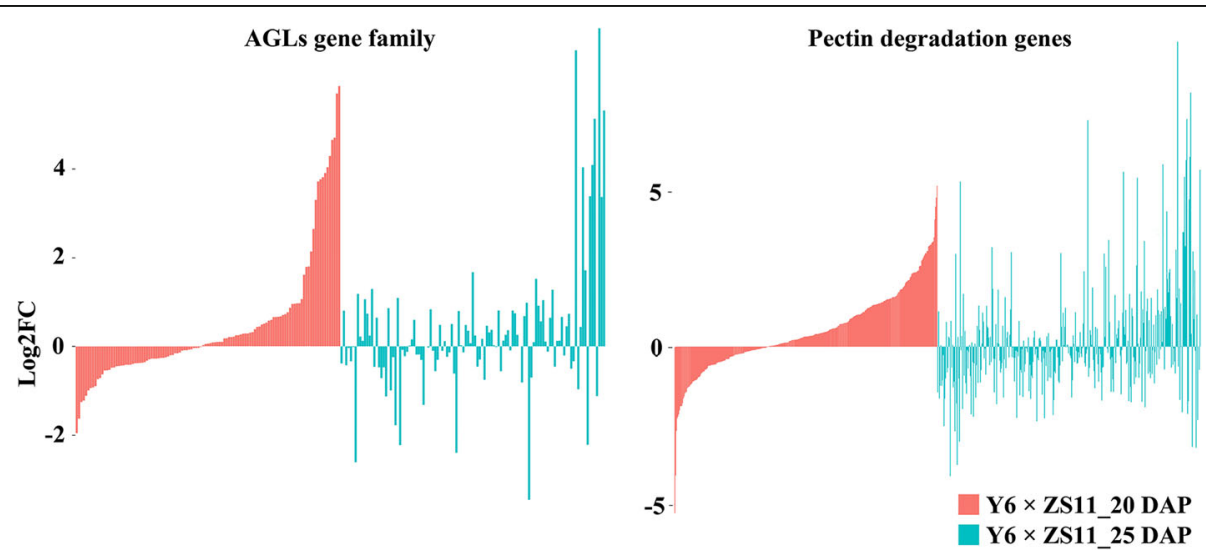

Fig. 8 Expression analysis of AGLS and pectin degradation pathway genes in reciprocal endosperm 
MEGs and 14 PEGs. The number of PEGs in B. napus was much less than MEGs, which is consistent with reports in A. thaliana, maize, sorghum and A. lyrata [29, 34, 54, 55]. The unbalanced MEGs and PEGs in plants agreed with the maternal-offspring co-adaptation theory, indicating the maternal genes were more favored during natural selection [56]. The different number of imprinted genes in 20 and 25 DAP rapeseed endosperm agreed with the previous report that imprinted genes varies with plant development. Xin et al. found the number of MEGs maximized in 10 DAP endosperm of maize, while the number of PEGs peaked in 7 DAP endosperm [57]. In wheat, $47.3 \%$ genes exhibited consistent imprinted expression pattern in 15, 20, and 25 DAP endosperm [47]. Besides, $40 \%$ genes were consistently imprinted in 10 and 12 DAP endosperm of maize [26, 54]. The percentage of consistently imprinted genes in this manuscript was much lower than that in wheat and maize. Since the imprinted genes in Brassica has not been well studied, identification of genome imprinting in more developmental stages of endosperm from different cultivars would be helpful to elaborate the imprinting characteristics in Brassica. Among the genes imprinted in both 20 and 25 DAP endosperm, we found BnaA03g05560D is a homolog of OsCWA1/BC1 that encodes a COBRA protein. OsCWA1/BC1 is important in secondary cell wall biosynthesis [58]. BnaA05g04610D is a homolog of AtPME17, which can change pectin physicochemical properties, induce the reduction of galacturonic acid to modify the cell wall architecture [59]. BnaC05g18500D is a homolog of AtPME6, which encodes a pectin methyl esterase that involved in pectin metabolism of cell wall, and embryo cell expansion and development [60, 61].

\section{Bias of genome imprinting in $A$ and $C$ subgenome of $B$. napus}

B. napus, as an allotetraploid could provide a model to determine whether genome imprinting exhibits expression bias between the homoeologous from $A$ and $C$ subgenome. Among the 297 imprinted genes in B. napus, we found 14 pairs of homologs, but only 8 homologous pairs were located on the A and C subgenome, respectively. As to the remaining 281 unpaired genes, 200 genes (71\%) were imprinted on the A subgenome and 81 genes (29\%) were imprinted on the C subgenome. This indicated that most of the imprinted genes were biased to A subgenome. During the polyploidization of B. napus, Li et al. found that the majority of gene pairs $(\sim 86.7 \%)$ in $B$. napus maintained their expression pattern in two diploid progenitors, and approximately $78.1 \%$ of the gene pairs showed expression bias with a preference toward the A subgenome [62]. Wu et al. also showed that $36.5 \%$ of the expressed gene pairs in resynthesized $B$. napus displayed expression bias with a slight preference toward the A genome [63]. Thus, we may suspect that the bias of imprinted gene number might be related to the expressional bias during rapeseed polyploidization.

\section{Genome imprinting is not conserved among different species}

Studies on Arabidopsis, rice, maize and other plants confirmed that imprinted genes are not conserved among plant species, only 21 imprinted genes were conserved between Arabidopsis and C. rubella, two genes were imprinted in both rice and Arabidopsis [28, 29]. In the present study, we found $15,2,5,3,10$, and 25 rapeseed imprinted genes with homologs imprinted in Arabidopsis, rice, castor bean, maize, B. rapa, and other $B$. napus lines, respectively [22-27]. The little overlap of imprinted genes among species, even between different lines of same species is common in plants, which might be due to the filtering of data analysis. In Arabidopsis, only 19 genes were imprinted both in Ler and Col-O accessions, while large majority of genes $(81 \%)$ were unique to a single study [64]. In the four available studies of maize, the majority of the imprinted genes (65\% MEGs and 41\% PEGs) were proposed by a single study, only 14 MEGs (8\%) and 23 PEGs (13\%) were commonly identified by all four studies [64]. Here, we only found 10 and 25 genes with orthologous imprinted in B. rapa and other B. napus lines (YN171 and 93275), respectively $[45,46]$. Among the conserved imprinted genes among species, BnaA06g38220D was found with homologs imprinted in maize and rice, which encodes an extensin-like protein and might be involved in lignin biosynthesis and interspecific reproductive disorder $[65,66]$. The homologs of BnaC07g22140D in A. thaliana (AT2G03210) encodes xyloglucan fucosyltransferase 2 (FUT2) that might be participated in cell wall organization [67]. BnaA05g08350D is a homolog of AtFIS2, which is important in repressing seed development before fertilization, and regulates embryo and endosperm development after the double fertilization $[16,68]$. In this study, we found 10 genes with homologs imprinted in $B$. rapa, which is a diploid parent of $B$. napus. These genes were annotated with functions in regulating DNA methylation (BnaA05g08770D and BnaA05g08350D) and cellulose synthesis (BnaA0826321D and BnaA08g26320D) (Additional file 6: Table S5; Additional file 7: Table S6). Hitherto, there is only one report of genome imprinting in 30 DAP endosperm of B. napus [46], and 25 imprinted genes $(8.42 \%)$ were overlapped with our study. These genes were annotated with functions in regulating ovule development (BnaA03g24210D), cell wall modification (BnaA04g24790D), apoptosis (BnaA10g23660D), and response to oxidative stress (BnaC09g54550D and BnaA09g31650D) (Additional file 6: Table S5; Additional file 7: Table S6). BnaA0826321D is a homolog of 
OsMYB61 and imprinted in B. rapa and other B. napus lines. In rice, OsNAC29/31 directly activate OsMYB61, which in turn activates the expression of cellulose synthase genes, and finally regulates secondary wall cellulose synthesis [69]. BnaA05g08770D, an overlapped imprinted gene with B. rapa, is a homolog of AtSUVH5. AtSUVH5 interacts with AtHDA19 and negatively regulates seed dormancy [70].

\section{The expressional pattern and clustering of imprinted genes in $B$. napus}

Previous researches reported that $80 \%$ of imprinted genes in Arabidopsis, 70\% in sorghum, and 78\% in rice were endosperm-specific or endosperm preferentially expressed genes. However, only $40 \%$ of imprinted genes in castor bean were endosperm-specific genes [24, 27, $29,34]$. In the present study, we found 26 imprinted genes were endosperm-specific expressed genes, while other genes were also expressed in different tissues of $B$. napus, indicating they might play functions throughout rapeseed development, not only in seed development.

In mammals, most of the imprinted genes are clustered on the chromosome [32, 33, 48]. In plants, only 28 imprinted genes were assigned to 12 clusters in sorghum, 77 genes in 33 clusters were identified in maize, and 7 imprinted genes in 3 clusters were reported in castor bean [26, 27, 29]. In rapeseed, we found 109 imprinted genes assigned to 35 clusters on chromosomes. The chromosome regions around these clusters might be imprinting control regions and could influence imprinted gene expression. We also found homologous gene pairs in these clusters, suggesting that genome duplication also made gene imprinting more complicate in rapeseed [34].

\section{Functions of imprinted genes in regulating endosperm development}

Imprinted genes have been confirmed with important roles in endosperm development $[40,50]$. Here we found the rapeseed imprinted genes were also enriched in process related to cell wall biosynthesis, and speculated that they might be related to the endosperm development. For example, BnaA03g05560D, which encodes irregular xylem 6 , was enriched in plant-type secondary cell wall biogenesis (GO: 0009834). Its homologous gene in rice is crucial for assembly of secondary cell wall [58]. BnaA01g03570D was enriched in cell wall organization (GO: 0071555), and the homolog in Arabidopsis was required for branch extension of xylan in cell wall [71]. In addition, most of the imprinted genes were enriched with binding and catalytic function (e.g. hydrolase and transferase activities). We also found imprinted genes enriched in biological processes, such as carbon metabolism, cellular metabolism, and biosynthesis of biological macromolecules. In Arabidopsis, triploid seed abortion in mutants of imprinted genes were related to the expression of genes involved in cell wall synthesis and degradation in endosperm [40,50]. Among the B. napus imprinted genes identified in this study, BnaA05g04610D, BnaC05g18500D and BnaA04g24790D were involved in pectin metabolism, which might be involved in endosperm cellularization. Besides, the expression changes of AGLs can hinder the formation and degradation of endosperm cell wall, and finally affect endosperm cellularization and lead to seed abortion. Here, we only found the expression of 5 AGLs was significantly changed in hybrid endosperm compared with parent endosperm. Of which, BnaA03g29530D (a homo$\log$ of AtAGL91) and BnaA09g16050D were significantly changed in 20 and 25 DAP endosperm of reciprocal hybrids. In Arabidopsis, the spatial-temporal expression of AGL91 was regulated by maternal siRNAs, and disruption or overexpression of $A G L 91$ in the endosperm altered seed size [72]. BnaA09g16050D encodes a pectin lyase-like superfamily protein, which might be a pectic substance that occurred as structural polysaccharides in the middle lamella and primary cell walls of higher plants [73]. BnaC04g24110D also encodes a pectin lyaselike superfamily protein, and its homologs in Arabidopsis (ADPG1) regulates lignin content and composition [74]. BnaA05g04610D is a homolog of AtPME17, which can change pectin physicochemical properties, induce the reduction of galacturonic acid to modify the cell wall architecture [75]. BnaAnng26700D is a homolog of OsFIE1, which is an essential member of polycomb repressive complex 2 (PRC2) that plays vital roles in early seed development through regulating endosperm cellularization and seed size [76, 77].

\section{Putative regulation of imprinted genes in B. napus}

TEs are important in driving plant genome expansion and species evolution, through influencing the genome structure (gene structure, inversion, translocation, and recombination) and gene expression [78-82]. It has been reported that TEs enriched in the vicinity of imprinted genes were extensively demethylated in endosperm, suggesting that TEs might be a driving force of genome imprinting [27]. In the present study, we also found significant enrichment of LTR/Copia and LTR/Gypsy TEs in the upstream and downstream of the imprinted genes. This was similar to castor bean, but the type of TEs enriched around imprinted genes of Arabidopsis, maize, and C. rubella is different $[26-28,34]$.

Genomic DNA methylation has also been taken as a driving force of genomic imprinting in plants, and most differentially methylated regions (DMRs) identified were hypomethylated in maternal alleles and hypermethylated in paternal alleles [26, 27]. However, only 11 imprinted 
genes $(0.06 \%)$ in $B$. napus were identified with DMRs, including one MEG confirmed with high GC methylation [46]. In the present study, DNA methylation analysis was not performed. But we correlate the imprinted genes with the miRNA regulation of B. napus [83], and found 12 imprinted genes might be targeted by miR158, miR171, miR160, miR399, miR394, and other six novel miRNAs. In Arabidopsis, functional loss of miR171 caused abnormal embryogenesis, and it was proved that a correct relationship between miR171 and HAM1 is necessary for normal embryogenesis [84]. miR160, miR171, miR394, and miR399 may participate in the early embryonic development and morphogenesis of maize and Arabidopsis, through transcriptional regulation of their target genes $[85,86]$. In addition, miR160 controls somatic embryogenesis induction by negatively regulating auxin-related genes (ARF10, ARF16, and ARF17) [87].

\section{Conclusions}

In the present study, we identified a total of 297 imprinted genes, including 283 MEGs and 14 PEGs in reciprocal hybrid endosperm, basing on the specific SNPs in two B. napus cultivars (Y6 and ZS11). Only 36 genes were continuously imprinted in 20 and 25 DAP endosperm. Besides, many imprinted genes in rapeseed were annotated to GO terms related to cell wall biosynthesis and endosperm cellularization. TEs analysis nearby the imprinted and non-imprinted genes revealed that LTR/Copia TEs were most enriched, indicating they might influence the expression pattern of imprinted genes. Moreover, the expression of 5 AGLs and 6 pectinrelated genes in hybrid endosperm were significantly changed when comparing with that in parent endosperm, which would be helpful to explain the normal developed reciprocal hybrid seeds. Generally, identification and characterization of imprinted genes in B. napus enriched the gene imprinting in dicotyledon plants, and provided a basis for further researches on how gene imprinting regulates seed development.

\section{Materials and methods}

\section{Tissue collection and RNA preparation}

Two B. napus L. cultivars (ZS11 and Y6) were provided by Jiangsu Institute of Agricultural Science in the Lixiahe District, and grown in the filed in Yangzhou, Jiangsu, China. Reciprocal crosses between two cultivars were carried out for F1 hybrids. The endosperm for RNA-seq was collected from immature seeds at 20 and 25 DAP, including the endosperm from reciprocal crosses and self-pollinated ZS11 and Y6. To avoid tissue contamination, the endosperm was sucked with an injector, which was inserted into the hole punched by a needle on the top of seeds (opposite to the embryo). Then, the endosperm was pooled and immediately stored in RNA extraction buffer [46]. Three biological replicates were included for each sample.

\section{RNA-seq analysis and data processing}

Total RNA was extracted by RNAprep pure Plant Kit (TIANGEN, China). The purity, concentration, and integrity of endosperm RNA were detected using agarose gel electrophoresis, Nanodrop, Qubit, and Agilent 2100. The eligible RNA samples were used for mRNA library construction and high-throughput sequencing on the Illumina Novaseq 6000 platform [88]. In total, 269.84 $\mathrm{Gb}$ and $268.73 \mathrm{~Gb}$ of raw data were generated from parents and reciprocal hybrids endosperm, respectively. The sequencing base qualities and reads qualities were assessed by FastQC-0.11.8 software (http://www. bioinformatics.babraham.ac.uk/projects/fastqc/). Lowquality and adaptor sequences in the raw data were eliminated using Trimmomatic-0.36 software (http:// www.usadellab.org/cms/uploads/supplementary/ Trimmomatic/) [89]. Then, the clean data were retained for further analysis of SNP and gene expression. The correlation coefficients among the three biological replicates of each sample were assessed for data reliability $(R>$ 0.95). The reads were mapped to the $B$. napus reference genome v4.1 (http://www.genoscope.cns.fr/brassicanapus/ data/) using Hisat2-2.1.0 software (https://ccb.jhu.edu/ software/hisat2/index.shtml) [90, 91], only two nucleotide mismatch was allowed in paired-end alignment. The mapping rate of all samples was $>90 \%$.

\section{SNP calling and identification of imprinted genes}

The RNA-seq data of ZS11 and Y6 were used for SNP screening. To increase the credibility, only the uniquely mapped reads were kept for analysis. SNP calling was performed using the Samtools-1.4 (http://samtools. sourceforge.net/) command Mpileup and the Bcftools program to identify the SNPs between ZS11 and Y6 $[92,93]$, and only the homozygous SNPs existed in at least two biological replicates and supported by $\geq 10$ reads in each library were retained as parental specific SNPs.

To identify the imprinted genes, the uniquely mapped reads from reciprocal hybrid endosperm were retained for SNP and expression analysis. The sequencing reads of hybrid endosperm containing maternal-derived or paternal-derived SNPs were identified and counted by Perl scripts. Theoretically, the ratio of maternal-derived allele to paternal-derived allele in the hybrid endosperm should be 2: 1 . We performed a two-tailed $\chi^{2}$ test on the ratio of maternal versus paternal allele counts for each

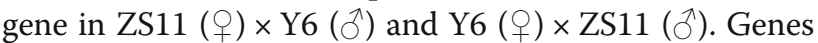
with parental allele bias deviated from 2: 1 in three biological replicates of both reciprocal hybrids were screened and defined as potential imprinted genes. We 
used stringent criteria to screen imprinted gene. Genes with a ratio of maternal-derived reads to paternalderived reads $\geq 10$ : 1 ( 5 times of maternal: paternal $=2: 1$, $\geq 90 \%$ maternally biased expression) in both reciprocal hybrids were taken as MEGs, while genes with a ratio of paternal-derived reads to maternal-derived reads $\geq 3: 2$ ( 3 times of paternal: maternal $=1: 2, \geq 60 \%$ paternally biased expression) were defined as PEGs. All the imprinted genes were screened with a threshold of $q<$ 0.05 .

\section{Reverse transcription and locus-specific sequencing}

RNA from the endosperm of parents and reciprocal hybrids were used for confirmation of imprinted genes. The cDNA was synthesized using HiScript III RT SuperMix for qPCR (Vazyme, China). Primers used for sequence amplification were designed with Primer Premier 5.0 and listed in Additional file 12: Table S11. The amplified fragments from parents and reciprocal hybrids were sequenced by Sanger sequencing (TsingKe Biological Technology, China), to confirm the existence of maternal or paternal specific SNPs in MEGs or PEGs.

\section{Gene expression analysis}

To investigate the expression pattern of imprinted genes in different tissues of $B$. napus, three biological replicates of root, stem, leaf, bud, and endosperm were collected for RNA-seq analysis. The sequencing data was mapped as mentioned above and the mapped reads were normalized using FPKM value. The heat map of imprinted genes in different tissues was generated based on the $\log _{10}$ transformed values of FPKM. The expression of imprinted and non-imprinted genes in parental endosperm were plotted with $\log _{2}$ FPKM.

\section{Gene ontology analysis}

GO enrichment was analyzed using OmicShare website (http://www.omicshare.com/tools/) and Blast2GO software (https://www.blast2go.com/) with a corrected $p<$ 0.05 . Samtools-1.4 was adopted for visualization of nucleic acid sequences based on the IDs of imprinted genes, and the output files were used for GO annotation with Blast2GO. Biomart tool (http://plants.ensembl.org/ biomart/martview/) was used for preparation of input files for Omicshare website.

\section{Clustering analysis}

For clustering analysis, we mapped 245 imprinted genes onto the $19 \mathrm{~B}$. napus chromosomes (except for 52 candidate imprinted genes located in Ann_random and Cnn random chromosomes) using MG2C (http://mg2c.iask. $\left.\mathrm{in} / \mathrm{mg} 2 \mathrm{c} \_\mathrm{v} 2.0 /\right)$. To analyze the gene distribution in chromosomes, sliding windows of $1 \mathrm{Mb}$ with step size $0.1 \mathrm{Mb}$ were used to compare the number of mapped reference genes and these imprinted genes were classified into clusters $(p<0.05)$ for further analysis.

\section{TE enrichment analysis nearby the imprinted genes} To verify the relationship between the imprinted genes and TEs, we used TransposonPSI software (https:// sourceforge.net/projects/transposonpsi/) and Bioperl:: SearchIO module (http://www.bioperl.org/wiki/ Installing_BioPerl) to analyze the type and number of TEs within $5 \mathrm{~kb}$ of the upstream and downstream of the imprinted genes and non-imprinted genes.

\section{Abbreviations \\ MEG: Maternal expressed gene; PEG: Paternal expressed gene; \\ TE: Transposable element; AGL: AGAMOUS-LIKE MADS-box gene; \\ ZS11: Zhongshuang 11; Y6: Yangyou 6; SNP: Single nucleotide \\ polymorphism; DAP: Days after pollination; FPKM: Fragments per kilobase per million; KEGG: Kyoto encyclopedia of genes and genomes; GO: Gene ontology; FUT2: Xyloglucan fucosyltransferase 2; DMR: Differentially methylated region}

\section{Supplementary Information}

The online version contains supplementary material available at https://doi. org/10.1186/s12870-021-02908-8.

Additional file 1: Fig. S1. Personal correlation coefficient analysis of three biological replicates of RNA-seq data.

Additional file 2: Table S1. SNPs between 20 DAP endosperm of Y6 and ZS11.

Additional file 3: Table S2. SNPs between 25 DAP endosperm of Y6 and ZS11.

Additional file 4: Table S3. The proportion of maternal and paternal reads in 20 DAP endosperm of reciprocal hybrids.

Additional file 5: Table S4. The proportion of maternal and paternal reads in 25 DAP endosperm of reciprocal hybrids.

Additional file 6: Table S5. Imprinted genes identified in 20 DAP endosperm of reciprocal hybrids.

Additional file 7: Table S6. Imprinted genes identified in 25 DAP endosperm of reciprocal hybrids.

Additional file 8: Table S7. Comparison of imprinted genes between 20 DAP and 25 DAP endosperm of reciprocal hybrids.

Additional file 9: Table S8. Tissue expressional pattern of imprinted genes (FPKM).

Additional file 10: Table S9. Cluster analysis of imprinted genes on $B$. napus chromosomes.

Additional file 11: Table S10. GO enrichment analysis of imprinted genes.

Additional file 12: Table S11. Primers used for validation of imprinted genes.

Acknowledgements

Not applicable.

Authors' contributions

$\mathrm{HR}$ performed the experiments and drafted the manuscript, $\mathrm{HR}, \mathrm{WY}$ and $\mathrm{BJ}$ sampled the plant materials. $\mathrm{HR}$ and $\mathrm{HZ}$ analyzed the sequencing data. JJ and $\mathrm{YW}$ revised the manuscript. All the authors approved the final manuscript.

\section{Funding}

This study was supported by the National Natural Science Foundations

(31771824, 31771825), the Natural Science Foundation of Jiangsu Province 
(BK20180101, BE2018356), the National Key Research and Development Program of China (2018YFE0108000), the Priority Academic Program Development of Jiangsu Higher Education Institutions. The founders did not play any roles in the design, analysis, interpretation of this study or relevant data.

\section{Availability of data and materials}

All the data pertaining to the present study have been included in the tables and figures of the manuscript, and the authors are pleased to share all the data and plant materials upon reasonable request.

\section{Declarations}

\section{Ethics approval and consent to participate}

This study has not directly involved humans or animals. No specific permission was required for use of these materials for experimental purposes. The seedlings were grown in the experimental field of Yangzhou University, Yangzhou, China as per standard practices, and samples were harvested at the required time. We comply with the Convention on the Trade in Endangered Species of Wild Fauna and Flora.

\section{Consent for publication}

Not applicable.

\section{Competing interests}

The authors declare that they have no competing interests.

\section{Author details}

1Jiangsu Provincial Key Laboratory of Crop Genetics and Physiology, Yangzhou University, Yangzhou 225009, China. ${ }^{2}$ Joint International Research Laboratory of Agriculture and Agri-Product Safety, the Ministry of Education of China, Yangzhou 225009, China.

Received: 12 March 2020 Accepted: 28 February 2021

Published online: 16 March 2021

\section{References}

1. Bauer MJ, Fischer RL. Genome demethylation and imprinting in the endosperm. Curr Opin in Plant Biol. 2011;14(2):162-7.

2. Frost JM, Moore GE. The importance of imprinting in the human placenta. PLoS Genet. 2010;6(7):e1001015.

3. Renfree MB, Suzuki S, Kaneko-Ishino T. The origin and evolution of genomic imprinting and viviparity in mammals. Philos T R Soc B. 2013;368(1609): 20120151.

4. Reik W, Walter J. Genomic imprinting: parental influence on the genome. Nat Rev Genet. 2001;2(1):21-32.

5. Feil R, Berger F. Convergent evolution of genomic imprinting in plants and mammals. Trends Genet. 2007;23(4):192-9.

6. Berger F, Hamamura Y, Ingouff M, Higashiyama T. Double fertilization caught in the act. Trends Plant Sci. 2008;13(8):437-43.

7. Gehring M. Genomic imprinting: insights from plants. Annu Rev Genet. 2013;47:187-208.

8. Li J, Berger F. Endosperm: food for humankind and fodder for scientific discoveries. New Phytol. 2012;195(2):290-305.

9. Raissig MT, Baroux C, Grossniklaus U. Regulation and flexibility of genomic imprinting during seed development. Plant Cell. 2011;23(1):16-26.

10. Moore T, Mills W. Evolutionary theories of imprinting - enough already! Adv Exp Med Biol. 2008;626:116-22.

11. Spencer HG, Clark AG. Non-conflict theories for the evolution of genomic imprinting. Heredity. 2014;113(2):112-8.

12. Kermicle JL. Dependence of the R-mottled aleurone phenotype in maize on mode of sexual transmission. Genetics. 1970;66(1):69-85.

13. Bartolomei MS, Zemel S, Tilghman SM. Parental imprinting of the mouse H19 gene. Nature. 1991;351(6322):153-5.

14. Barton SC, Surani MA, Norris ML. Role of paternal and maternal genomes in mouse development. Nature. 1984;311(5984):374-6.

15. Grossniklaus U, Vielle-Calzada JP, Hoeppner MA, Gagliano WB. Maternal control of embryogenesis by MEDEA, a polycomb group gene in Arabidopsis. Science. 1998;280(5362):446-50.

16. Luo M, Bilodeau P, Dennis ES, Peacock WJ, Chaudhury A. Expression and parent-of-origin effects for FIS2, MEA, and FIE in the endosperm and embryo of developing Arabidopsis seeds. Proc Natl Acad Sci U S A. 2000;97(19): $10637-42$.

17. Gutierrez-Marcos JF, Pennington PD, Costa LM, Dickinson HG. Imprinting in the endosperm: a possible role in preventing wide hybridization. Philos T R Soc B. 2003;358(1434):1105-11.

18. Gutierrez-Marcos JF, Costa LM, Biderre-Petit C, Khbaya B, O'Sullivan DM, Wormald M, Perez P. Dickinson HG: maternally expressed gene1 is a novel maize endosperm transfer cell-specific gene with a maternal parent-oforigin pattern of expression. Plant Cell. 2004;16(5):1288-301.

19. Hermon P, Srilunchang KO, Zou J, Dresselhaus T, Danilevskaya ON. Activation of the imprinted Polycomb Group Fiel gene in maize endosperm requires demethylation of the maternal allele. Plant Mol Biol. 2007;64(4): 387-95.

20. Huh JH, Bauer MJ, Hsieh TF, Fischer RL. Cellular programming of plant gene imprinting. Cell. 2008;132(5):735-44.

21. Jullien PE, Berger F. Gamete-specific epigenetic mechanisms shape genomic imprinting. Curr Opin Plant Biol. 2009;12(5):637-42.

22. Gehring M, Missirian V, Henikoff S. Genomic analysis of parent-of-origin allelic expression in Arabidopsis thaliana seeds. PLoS One. 2011;6(8):e23687.

23. Hsieh TF, Shin J, Uzawa R, Silva P, Cohen S, Bauer MJ, Hashimoto M, Kirkbride RC, Harada JJ, Zilberman D, et al. Regulation of imprinted gene expression in Arabidopsis endosperm. Proc Natl Acad Sci U S A. 2011;108(5): 1755-62.

24. Luo M, Taylor JM, Spriggs A, Zhang H, Wu X, Russell S, Singh M, Koltunow A. A genome-wide survey of imprinted genes in rice seeds reveals imprinting primarily occurs in the endosperm. PLoS Genet. 2011;7(6): e1002125.

25. Waters AJ, Makarevitch I, Eichten SR, Swanson-Wagner RA, Yeh CT, Xu W, Schnable PS, Vaughn MW, Gehring M, Springer NM. Parent-of-origin effects on gene expression and DNA methylation in the maize endosperm. Plant Cell. 2011;23(12):4221-33.

26. Zhang M, Zhao H, Xie S, Chen J, Xu Y, Wang K, Zhao H, Guan H, Hu X, Jiao $Y$, et al. Extensive, clustered parental imprinting of protein-coding and noncoding RNAs in developing maize endosperm. Proc Natl Acad Sci U S A 2011;108(50):20042-7.

27. Xu W, Dai M, Li F, Liu A. Genomic imprinting, methylation and parent-oforigin effects in reciprocal hybrid endosperm of castor bean. Nucleic Acid Res. 2014:42(11):6987-98.

28. Hatorangan MR, Laenen B, Steige KA, Slotte T, Kohler C. Rapid evolution of genomic imprinting in two species of the Brassicaceae. Plant Cell. 2016; 28(8):1815-27.

29. Zhang M, Li N, He W, Zhang H, Yang W, Liu B. Genome-wide screen of genes imprinted in sorghum endosperm, and the roles of allelic differential cytosine methylation. Plant J. 2016;85(3):424-36.

30. Stoute Al, Varenko V, King GJ, Scott RJ, Kurup S. Parental genome imbalance in Brassica oleracea causes asymmetric triploid block. Plant J. 2012;71(3):503-16.

31. Zhang HY, Luo M, Johnson SD, Zhu XW, Liu L, Huang F, Liu YT, Xu PZ, Wu XJ. Parental genome imbalance causes post-zygotic seed lethality and deregulates imprinting in rice. Rice. 2016;9(1):43.

32. Yang $T$, Adamson TE, Resnick JL, Leff $S$, Wevrick R, Francke U, Jenkins NA, Copeland NG, Brannan Cl. A mouse model for Prader-Willi syndrome imprinting-Centre mutations. Nat Genet. 1998;19(1):25-31.

33. Bartolomei MS, Ferguson-Smith AC. Mammalian genomic imprinting. CSH Perspect Biol. 2011;3(7):839-48.

34. Wolff $P$, Weinhofer I, Seguin J, Roszak P, Beisel C, Donoghue MT, Spillane C, Nordborg M, Rehmsmeier M, Kohler C. High-resolution analysis of parent-oforigin allelic expression in the Arabidopsis endosperm. PLoS Genet. 2011; 7(6):e1002126

35. Sha K. A mechanistic view of genomic imprinting. Annu Rev Genom Hum G. 2008:9:197-216.

36. Gehring M, Bubb KL, Henikoff S. Extensive demethylation of repetitive elements during seed development underlies gene imprinting. Science. 2009;324(5933):1447-51.

37. Zemach A, Kim MY, Silva P, Rodrigues JA, Dotson B, Brooks MD, Zilberman D. Local DNA hypomethylation activates genes in rice endosperm. Proc Natl Acad Sci U S A. 2010;107(43):18729-34.

38. Petit M, Guidat C, Daniel J, Denis E, Montoriol E, Bui QT, Lim KY, Kovarik A, Leitch AR, Grandbastien MA, et al. Mobilization of retrotransposons in synthetic allotetraploid tobacco. New Phytol. 2010;186(1):135-47.

39. Wang $N$, Wang $H$, Wang $H$, Zhang D, Wu Y, Ou X, Liu S, Dong Z, Liu B. Transpositional reactivation of the dart transposon family in rice lines 
derived from introgressive hybridization with Zizania latifolia. BMC Plant Biol. 2010;10:190.

40. Wolff P, Jiang H, Wang G, Santos-Gonzalez J, Kohler C. Paternally expressed imprinted genes establish postzygotic hybridization barriers in Arabidopsis thaliana. elife. 2015;4.

41. Kim CK, Seol YJ, Perumal S, Lee J, Waminal NE, Jayakodi M, Lee SC, Jin S, Choi BS, Yu Y, et al. Re-exploration of U's triangle Brassica species based on chloroplast genomes and 45S nrDNA sequences. Sci Rep. 2018;8(1):7353.

42. Wittkop B, Snowdon RJ, Friedt W. New NIRS calibrations for fiber fractions reveal broad genetic variation in Brassica napus seed quality. J Agric Food Chem. 2012;60(9):2248-56.

43. Borisjuk L, Neuberger T, Schwender J, Heinzel N, Sunderhaus S, Fuchs J, Hay JO, Tschiersch H, Braun HP, Denolf P, et al. Seed architecture shapes embryo metabolism in oilseed rape. Plant Cell. 2013;25(5):1625-40.

44. Sreenivasulu N, Wobus U. Seed-development programs: a systems biologybased comparison between dicots and monocots. Annu Rev Plant Biol. 2013;64:189-217.

45. Yoshida T, Kawanabe T, Bo Y, Fujimoto R, Kawabe A. Genome-wide analysis of parent-of-origin allelic expression in endosperms of Brassicaceae species, Brassica rapa. Plant Cell Physiol. 2018;59(12):2590-601.

46. Liu J, Li J, Liu HF, Fan SH, Singh S, Zhou XR, Hu ZY, Wang HZ, Hua W. Genome-wide screening and analysis of imprinted genes in rapeseed (Brassica napus L.) endosperm. DNA Res. 2018;25(6):629-40.

47. Yang G, Liu Z, Gao L, Yu K, Feng M, Yao Y, Peng H, Hu Z, Sun Q, Ni Z, et al. Genomic imprinting was evolutionarily conserved during wheat polyploidization. Plant Cell. 2018;30(1):37-47.

48. Barlow DP. Genomic imprinting: a mammalian epigenetic discovery model. Annu Rev Genet. 2011;45:379-403.

49. Hsieh TF, Ibarra CA, Silva P, Zemach A, Eshed-Williams L, Fischer RL, Zilberman D. Genome-wide demethylation of Arabidopsis endosperm. Science. 2009;324(5933):1451-4.

50. Kradolfer $D$, Wolff $P$, Jiang $H$, Siretskiy A, Kohler C. An imprinted gene underlies postzygotic reproductive isolation in Arabidopsis thaliana. Dev Cell. 2013;26(5):525-35.

51. Costa LM, Yuan J, Rouster J, Paul W, Dickinson H, Gutierrez-Marcos JF. Maternal control of nutrient allocation in plant seeds by genomic imprinting. Curr Biol. 2012;22(2):160-5.

52. Kinoshita T, Yadegari R, Harada JJ, Goldberg RB, Fischer RL. Imprinting of the MEDEA polycomb gene in the Arabidopsis endosperm. Plant Cell. 1999; 11(10):1945-52.

53. Kinoshita T, Miura A, Choi Y, Kinoshita Y, Cao X, Jacobsen SE, Fischer RL, Kakutani T. One-way control of FWA imprinting in Arabidopsis endosperm by DNA methylation. Science. 2004;303(5657):521-3.

54. Dong X, Zhang M, Chen J, Peng L, Zhang N, Wang X, Lai J. Dynamic and antagonistic allele-specific epigenetic modifications controlling the expression of imprinted genes in maize endosperm. Mol Plant. 2017;10(3):442-55.

55. Klosinska M, Picard CL, Gehring M. Conserved imprinting associated with unique epigenetic signatures in the Arabidopsis genus. Nat Plant. 2016;2:16145.

56. Wolf JB, Hager R. A maternal-offspring coadaptation theory for the evolution of genomic imprinting. PLoS Biol. 2006;4(12):e380.

57. Xin M, Yang R, Li G, Chen H, Laurie J, Ma C, Wang D, Yao Y, Larkins BA, Sun $Q$, et al. Dynamic expression of imprinted genes associates with maternally controlled nutrient allocation during maize endosperm development. Plant Cell. 2013;25(9):3212-27.

58. Sato $K$, Ito $S$, Fujii $T$, Suzuki $R$, Takenouchi $S$, Nakaba $S$, Funada $R$, Sano $Y$, Kajita S, Kitano H, et al. The carbohydrate-binding module (CBM)-like sequence is crucial for rice CWA1/BC1 function in proper assembly of secondary cell wall materials. Plant Signal Behav. 2010;5(11):1433-6.

59. Roig-Oliver M, Rayon C, Roulard R, Fournet F, Bota J, Flexas J. Reduced photosynthesis in Arabidopsis thaliana atpme17.2 and atpae11.1 mutants is associated to altered cell wall composition. Physiol Plantarum. 2020. https:// onlinelibrary.wiley.com/doi/epdf/10.1111/ppl.13186.

60. Wang M, Yuan D, Gao W, Li Y, Tan J, Zhang X. A comparative genome analysis of PME and PMEl families reveals the evolution of pectin metabolism in plant cell walls. PLoS One. 2013;8(8):e72082.

61. Levesque-Tremblay G, Muller K, Mansfield SD, Haughn GW. HIGHLY METHYL ESTERIFIED SEEDS is a pectin methyl esterase involved in embryo development. Plant Physiol. 2015;167(3):725-37.

62. Li M, Wang R, Wu X, Wang J. Homoeolog expression bias and expression level dominance (ELD) in four tissues of natural allotetraploid Brassica napus. BMC Genomics. 2020;21(1):330.
63. Wu J, Lin L, Xu M, Chen P, Liu D, Sun Q, Ran L, Wang Y. Homoeolog expression bias and expression level dominance in resynthesized allopolyploid Brassica napus. BMC Genomics. 2018;19(1):586.

64. Wyder S, Raissig MT, Grossniklaus U. Consistent reanalysis of genome-wide imprinting studies in plants using generalized linear models increases concordance across datasets. Sci Rep. 2019;9(1):1320-32.

65. Alves CML, Noyszewski AK, Smith AG. Structure and function of class III pistil-specific extensin-like protein in interspecific reproductive barriers. BMC Plant Biol. 2019;19(1):118.

66. Ke S, Luan X, Liang J, Hung YH, Hsieh TF, Zhang XQ. Rice OsPEX1, an extensin-like protein, affects lignin biosynthesis and plant growth. Plant Mol Biol. 2019;100(1-2):151-61.

67. Sarria R, Wagner TA, O'Neill MA, Faik A, Wilkerson CG, Keegstra K, Raikhel NV. Characterization of a family of Arabidopsis genes related to xyloglucan fucosyltransferase1. Plant Physiol. 2001;127(4):1595-606.

68. Wang D, Tyson MD, Jackson SS, Yadegari R. Partially redundant functions of two SET-domain polycomb-group proteins in controlling initiation of seed development in Arabidopsis. Proc Natl Acad Sci U S A. 2006;103(35):13244-9.

69. Huang DB, Wang SG, Zhang BC, Shang-Guan KK, Shi YY, Zhang DM, Liu XL, Wu K, Xu ZP, Fu XD, et al. A gibberellin-mediated DELLA-NAC signaling cascade regulates cellulose synthesis in rice. Plant Cell. 2015;27(6):1681-96.

70. Zhou Y, Yang P, Zhang F, Luo X, Xie J. Histone deacetylase HDA19 interacts with histone methyltransferase SUVH5 to regulate seed dormancy in Arabidopsis. Plant Biol. 2020.

71. Mortimer JC, Miles GP, Brown DM, Zhang Z, Segura MP, Weimar T, Yu X, Seffen KA, Stephens E, Turner SR, et al. Absence of branches from xylan in Arabidopsis gux mutants reveals potential for simplification of lignocellulosic biomass. Proc Natl Acad Sci U S A. 2010;107(40):17409-14.

72. Kirkbride RC, Lu J, Zhang CQ, Mosher RA, Baulcombe DC, Chen ZI. Maternal small RNAs mediate spatial-temporal regulation of gene expression, imprinting, and seed development in Arabidopsis. Proc Natl Acad Sci U S A. 2019;116(17):8633.

73. Yadav S, Yadav PK, Yadav D, Yadav KDS. Pectin lyase: a review. Process Biochem. 2009;44(1):1-10.

74. Gallego-Giraldo L, Liu C, Pose-Albacete S, Pattathil S, Peralta AG, Young J, Westpheling J, Hahn MG, Rao XL, Knox JP, et al. Arabidopsis DEHISCENCE ZONE POLYGALACTURONASE 1 (ADPG1) releases latent defense signals in stems with reduced lignin content. Proc Natl Acad Sci U S A. 2020;117(6):3281-90.

75. Senechal F, Graff L, Surcouf O, Marcelo P, Rayon C, Bouton S, Mareck A, Mouille $\mathrm{G}$, Stintzi A, Hofte $\mathrm{H}$, et al. Arabidopsis PECTIN METHYLESTERASE17 is co-expressed with and processed by SBT3.5, a subtilisin-like serine protease. Ann Bot. 2014;114(6):1161-75.

76. Folsom JJ, Begcy K, Hao X, Wang D, Walia H. Rice fertilization-independent Endosperm 1 regulates seed size under heat stress by controlling early endosperm development. Plant Physiol. 2014;165(1):238-48.

77. Cheng X, Pan M. E Z, Zhou Y, Niu B, Chen C: functional divergence of two duplicated Fertilization Independent Endosperm genes in rice with respect to seed development. Plant J. 2020;104(1):124-37.

78. Mc CB. The origin and behavior of mutable loci in maize. Proc Natl Acad Sci U S A. 1950;36(6):344-55.

79. Feschotte C. Transposable elements and the evolution of regulatory networks. Nat Rev Genet. 2008;9(5):397-405.

80. Fedoroff NV. Presidential address. Transposable elements, epigenetics, and genome evolution. Science. 2012;338(6108):758-67.

81. Lisch D. Regulation of the Mutator system of transposons in maize. Methods Mol Biol. 2013;1057:123-42.

82. Bennetzen $\mathrm{JL}$, Wang $\mathrm{H}$. The contributions of transposable elements to the structure, function, and evolution of plant genomes. Annu Rev Plant Biol. 2014:65:505-30.

83. Shen E, Zou J, Hubertus Behrens F, Chen L, Ye C, Dai S, Li R, Ni M, Jiang X, Qiu J, et al. Identification, evolution, and expression partitioning of miRNAs in allopolyploid Brassica napus. J Exp Bot. 2015;66(22):7241-53.

84. Takanashi H, Sumiyoshi H, Mogi M, Hayashi Y, Ohnishi T. Tsutsumi N: miRNAs control HAM1 functions at the single-cell-layer level and are essential for normal embryogenesis in Arabidopsis. Plant Mol Biol. 2018;96(6):627-40.

85. Armenta-Medina A, Lepe-Soltero D, Xiang D, Datla R, Abreu-Goodger C, Gillmor CS. Arabidopsis thaliana miRNAs promote embryo pattern formation beginning in the zygote. Dev Biol. 2017;431(2):145-51.

86. Li D, Liu Z, Gao L, Wang L, Gao M, Jiao Z, Qiao H, Yang J, Chen M, Yao L, et al. Genome-wide identification and characterization of microRNAs in developing grains of Zea mays L. PLoS One. 2016;11(4):e0153168. 
87. Wojcik AM, Nodine MD. Gaj MD: miR160 and miR166/165 contribute to the LEC2-mediated auxin response involved in the somatic embryogenesis induction in Arabidopsis. Front Plant Sci. 2017;8:2024.

88. Jiang J, Zhu S, Yuan Y, Wang Y, Zeng L, Batley J, Wang YP. Transcriptomic comparison between developing seeds of yellow- and black-seeded Brassica napus reveals that genes influence seed quality. BMC Plant Biol. 2019;19(1):203.

89. Bolger AM, Lohse M, Usadel B. Trimmomatic: a flexible trimmer for Illumina sequence data. Bioinformatics. 2014;30(15):2114-20.

90. Chalhoub B, Denoeud F, Liu S, Parkin IA, Tang H, Wang X, Chiquet J, Belcram H, Tong C, Samans B, et al. Early allopolyploid evolution in the post-Neolithic Brassica napus oilseed genome. Science. 2014;345(6199):950-3.

91. Kim D, Langmead B, Salzberg SL. HISAT: a fast spliced aligner with low memory requirements. Nat Methods. 2015;12(4):357-60.

92. Li H, Handsaker B, Wysoker A, Fennell T, Ruan J, Homer N, Marth G, Abecasis $G$, Durbin R. The sequence alignment/map format and SAMtools. Bioinformatics. 2009;25(16):2078-9.

93. Li H. A statistical framework for SNP calling, mutation discovery, association mapping and population genetical parameter estimation from sequencing data. Bioinformatics. 2011;27(21):2987-93.

\section{Publisher's Note}

Springer Nature remains neutral with regard to jurisdictional claims in published maps and institutional affiliations.

Ready to submit your research? Choose BMC and benefit from:

- fast, convenient online submission

- thorough peer review by experienced researchers in your field

- rapid publication on acceptance

- support for research data, including large and complex data types

- gold Open Access which fosters wider collaboration and increased citations

- maximum visibility for your research: over $100 \mathrm{M}$ website views per year

At BMC, research is always in progress.

Learn more biomedcentral.com/submissions 